ARTICLE

DOI: 10.1038/s41467-017-00659-5 OPEN

\title{
Squirming motion of baby skyrmions in nematic fluids
}

Paul J. Ackerman ${ }^{1,2}$, Timothy Boyle ${ }^{1}$ \& Ivan I. Smalyukh (1) 1,2,3,4

Skyrmions are topologically protected continuous field configurations that cannot be smoothly transformed to a uniform state. They behave like particles and give origins to the field of skyrmionics that promises racetrack memory and other technological applications. Unraveling the non-equilibrium behavior of such topological solitons is a challenge. We realize skyrmions in a chiral liquid crystal and, using numerical modeling and polarized video microscopy, demonstrate electrically driven squirming motion. We reveal the intricate details of non-equilibrium topology-preserving textural changes driving this behavior. Direction of the skyrmion's motion is robustly controlled in a plane orthogonal to the applied field and can be reversed by varying frequency. Our findings may spur a paradigm of soliton dynamics in soft matter, with a rich interplay between topology, chirality, and orientational viscoelasticity.

\footnotetext{
${ }^{1}$ Department of Physics, University of Colorado, Boulder, Colorado 80309, USA. ${ }^{2}$ Department of Electrical, Computer, and Energy Engineering, University of Colorado, Boulder, Colorado 80309, USA. ${ }^{3}$ Soft Materials Research Center and Materials Science and Engineering Program, University of Colorado, Boulder, Colorado 80309, USA. ${ }^{4}$ Renewable and Sustainable Energy Institute, National Renewable Energy Laboratory and University of Colorado, Boulder, Colorado 80309, USA. Correspondence and requests for materials should be addressed to I.I.S. (email: ivan.smalyukh@colorado.edu)
} 
$\mathrm{M}$ otion of topological defects enables the plasticity of metals and mediates condensed matter phase transitions, as well as is responsible for numerous irreversible processes in physical systems ranging from cosmology to elementary particles and condensed matter ${ }^{1}$. Topological defects enable the existence of thermodynamically stable condensed matter phases, such as the twist grain boundary and blue phases of liquid crystals (LCs) ${ }^{1}$. However, apart from such exotic condensed phases that exhibit topological defects in the ground state, singular defects tend to annihilate and typically exist within transient short-lived processes. In soft active matter ${ }^{2}$, where weak external stimuli or local energy conversion fuel strong localized responses, unexpected recent findings include activity-driven generation and propulsion of the singular defects, spatially localized regions where order cannot be defined ${ }^{2-5}$. Active matter defects continuously progress through the cycles of generation and annihilation, displaying directional motion and other unexpected dynamics ${ }^{3-5}$. In chiral ferromagnetic solids, on the other hand, much of the recent interest is attracted by nonsingular solitonic two-dimensional (2D) topological defects ${ }^{6-12}$, which are often called 'baby skyrmions' because they are low-dimensional topological counterparts of the higher-dimensional Skyrme solitons originally used to model elementary particles with different baryon numbers ${ }^{13}$. These baby skyrmions can be stable both as individual particle-like excitations ${ }^{\gamma}, 11,12$ and as building blocks of thermodynamically stable phases, such as the so-called 'A-phase' ${ }^{6,8}$. Furthermore, electric currents induce directional motion of such solitonic defects in solid films ${ }^{11}$, which is of interest for a new breed of racetrack memory devices and other skyrmionics applications ${ }^{12}$. However, the experimental study of field-induced dynamics of baby skyrmions presents a challenge because techniques capable of imaging spin textures and field configurations lack the needed simultaneous spatial and temporal resolution $^{6-12}$.

In this work, we realize baby skyrmions in chiral nematic LCs, which were previously studied only as static field configurations $\mathrm{s}^{14}, 15$, and we show that low voltages can drive motion of such topological defects in directions orthogonal to the applied electric field. The chirality-stabilized skyrmions swim like defects in active matter ${ }^{2-5}$, albeit never annihilating and being driven by modulation of applied voltage rather than chemical energy conversion. At the same time, similar to the skyrmions in ferromagnetic solids ${ }^{11,12}$, these skyrmions move without relying on fluid flows. Enabled mainly by rotational dynamics of the LC molecular alignment field, this low-Reynolds-number translational motion of baby skyrmions stems from electrically driven squirming of their localized twisted regions. Though these topology-protected twisted regions have no membrane or physical interface, periodic relaxation and tightening of the twisted region make the skyrmions expand, contract and morph as they move, in this aspect resembling squirming motion ${ }^{16,17}$. Applied voltage driving schemes control both the direction and speed of these topological defects uniformly throughout the sample. Using a combination of laser tweezers, video microscopy, three-dimensional nonlinear optical imaging and numerical modeling ${ }^{14,18}$, we uncover the underlying physical mechanisms and reveal connections to the intrinsic chirality and orientational viscoelasticity of the LC. The controlled motion of nonannihilating topological solitons may enable versatile forms of particle transport and dynamic memory devices ${ }^{12}$, and may potentially spur a paradigm of out-of-equilibrium solitonic matter in LC systems ${ }^{2-5}$.

\section{Results}

Experimental realization and numerical modeling. We use a chiral nematic LC that has an intrinsic tendency for its local average rod-like molecular orientation $\mathbf{n}$, dubbed 'director', to twist around a helical axis orthogonal to $\mathbf{n}^{1}$, where $2 \pi$ rotation of n defines a spatial periodicity called 'helicoidal pitch' $p$. Our lefthanded chiral LC is a nematic host MLC-6609 doped with a chiral additive ZLI-811 (both from Merck), with a negative dielectric anisotropy, such that $\mathbf{n}(\mathbf{r})$ tends to orient orthogonally to the applied electric field. The LC is confined in a glass cell of thickness $d \approx p$ with substrates treated to produce perpendicular boundary conditions for $\mathbf{n}$. This confinement is incompatible with the helicoidal configuration of the chiral nematic LC and prompts unwinding of the director to a uniform vertically aligned state, as well as the formation of localized $\mathbf{n}(\mathbf{r})$-configurations that embed energetically favorable twist while conforming to the uniform vertical far-field background $\mathbf{n}_{0}$ and the vertical surface boundary conditions ${ }^{14,15}$. Figure 1 illustrates the geometry of our experiments, in which the skyrmion is oriented orthogonally to confining substrates and along the electric field applied to the transparent electrodes at the inner surfaces of the confining glass plates. When alternating current (AC) voltage with frequency $f_{c}=1-10 \mathrm{kHz}$ is applied to the electrodes, because of the LC's negative dielectric anisotropy, the director around the skyrmion rotates from its initial vertical orientation (Fig. 1a) to an in-plane orientation (Fig. 1b), whereas the skyrmion morphs its structure while staying oriented orthogonally to confining substrates. The direction of in-plane orientation of $\mathbf{n}_{0}$ in the cell midplane far away from skyrmions is either selected spontaneously as a result of a process (typically 5-10 s long) involving coarsening of domains that follows voltage application, or it can be predetermined by inducing a small pretilt with respect to the perpendicular surface boundary conditions, as detailed in the
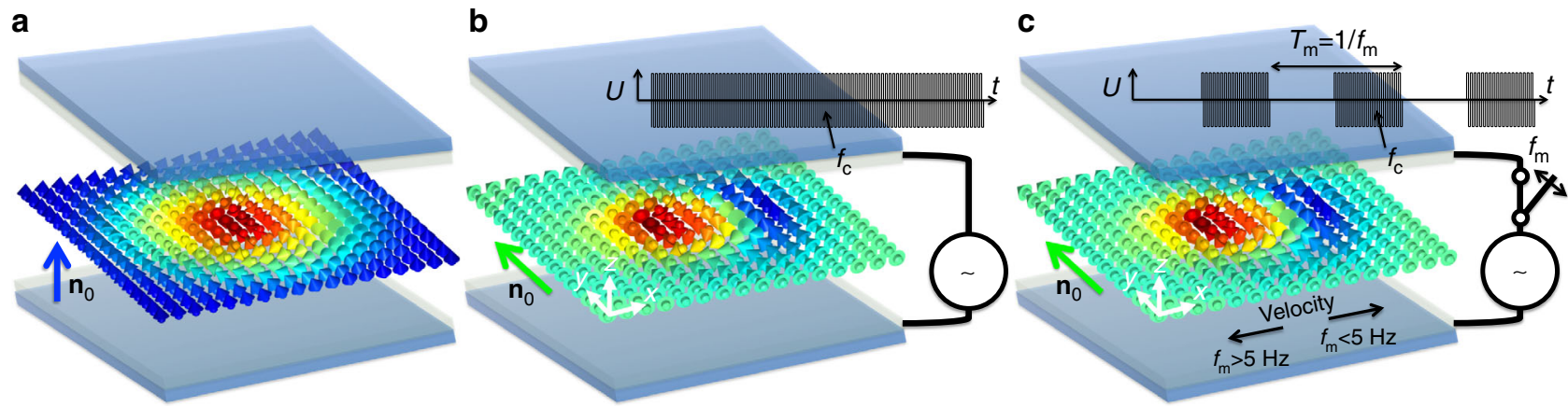

Fig. 1 Schematic illustration of experimental geometry. a A skyrmion oriented with its axis orthogonal to confining substrates at no applied fields. b Electric field with frequency $f_{c}=1-10 \mathrm{kHz}$ and amplitude, $U$, schematically illustrated as a function of time, $t$, applied across the sample thickness rotates the farfield director $\mathbf{n}_{0}$ away from the skyrmion axis and normal to the sample's confining substrates to be in-plane and morphs the skyrmionic structure. $\mathbf{c}$ When this electric field with frequency $f_{\mathrm{c}}=1-10 \mathrm{kHz}$ is additionally modulated with the modulation frequency $f_{\mathrm{m}}$, the skyrmion moves in a direction determined by $f_{\mathrm{m}}$ 

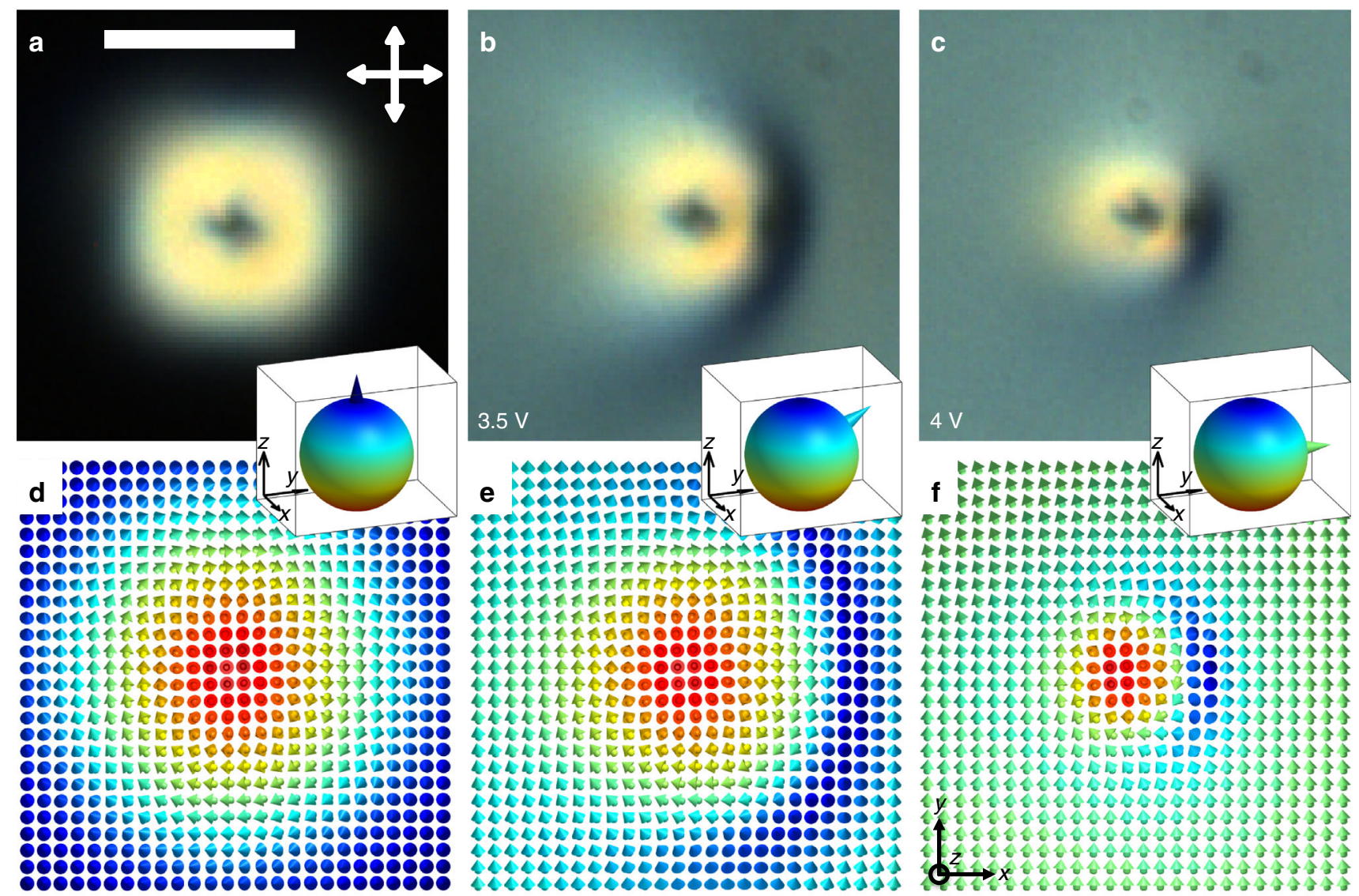

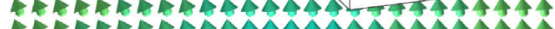

-

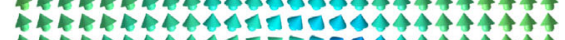

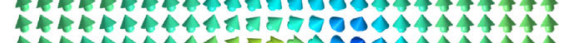

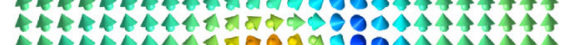

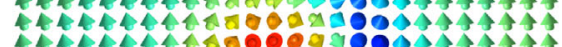

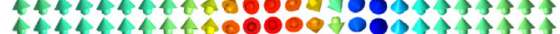

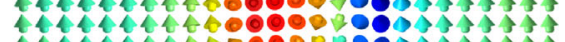

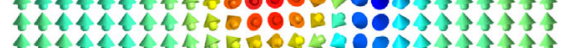

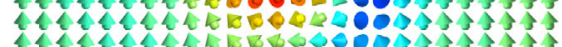

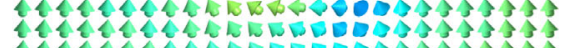

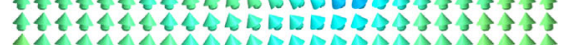
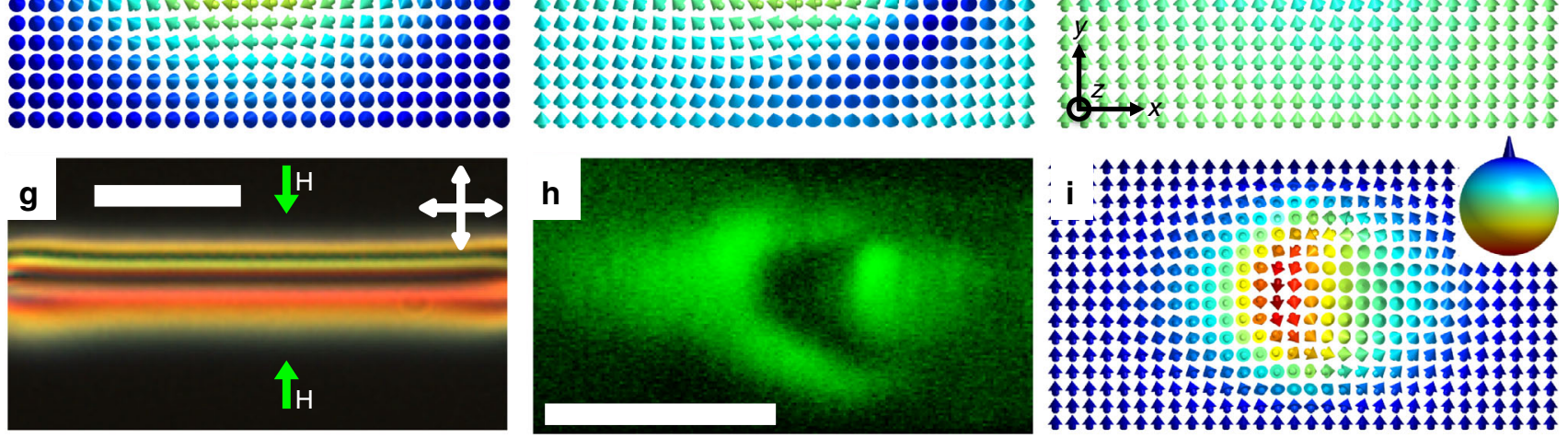

Fig. 2 Topology and electric switching of baby skyrmions. a-c POM micrographs of a skyrmion at (a) no applied fields and (b, c) at voltages indicated on images, the scale bar is $10 \mu \mathrm{m}$. Crossed polarizers used to obtain POM micrographs $(\mathbf{a}-\mathbf{c})$ are shown using white double arrows in $(\mathbf{a})$. $f_{\mathrm{c}}=1 \mathrm{kHz}$. d-f Computer-simulated vectorized $\mathbf{n}(\mathbf{r})$-configurations corresponding to $(\mathbf{a}-\mathbf{c})$, where $\mathbf{n}(\mathbf{r})$ is shown using arrows colored according to their orientations and corresponding points on the $S^{2}$-sphere (insets), with the orientation of $\mathbf{n}_{0}$ on $S^{2}$ shown using cones. $\mathbf{g}$ POM micrograph of a baby skyrmion in a glass cell with perpendicular boundary conditions. The scale bar is $20 \mu \mathrm{m}$. h Polarized nonlinear optical image of the skyrmion's $2 \mathrm{D}$ plane containing $\mathbf{n}_{\mathrm{O}}$. The image was obtained in the vertical cross-section along the $\mathrm{H}-\mathrm{H}$ line of $\mathbf{g}$. The scale bar is $10 \mu \mathrm{m}$. $\mathbf{i}$ The computer-simulated $\mathbf{n}(\mathbf{r})$ corresponding to (h); computer-simulated analogs of images $(\mathbf{g}, \mathbf{h})$ are shown in the Supplementary Fig. 1

methods section. When applied voltage is additionally modulated with lower modulation frequency $f_{\mathrm{m}}<f_{\mathrm{c}}$ (Fig. 1c), the skyrmion translates in a lateral direction orthogonal to $\mathbf{n}_{0}$ that depends on $f_{\mathrm{m}}$ (along one of the black arrows depicted in Fig. 1c). The design of our voltage-driving scheme with high $f_{\mathrm{c}}$ allows us to preclude complex hydrodynamic effects ${ }^{1}$ that could be associated with the response of ionic impurities to low-frequency electric fields, which assures purely dielectric response of the LC to fields and is key for elucidating the physical mechanism behind the studied skyrmion dynamics.

Figure $2 \mathrm{a}$ shows an axially symmetric solitonic baby skyrmion field configuration, as viewed using polarizing optical microscopy (POM) between crossed polarizers, with the corresponding results of detailed numerical modeling and nonlinear optical imaging $^{18,19}$ presented in Figs. 3, 4, respectively. A spontaneous formation of these solitons occurs during cooling of the sample through the isotropic-nematic phase transition, albeit they can be also controllably generated using laser tweezers ${ }^{14}$, 15 . With increasing the applied voltage above a well defined threshold $\approx 2 \mathrm{~V}$, the director far away from the baby skyrmion transitions from the initial vertical to tilted and then in-plane orientation. This director reorientation in response to the applied field is similar to that in LC displays ${ }^{20}$. This rotation morphs $\mathbf{n}(\mathbf{r})$ of the localized solitonic structure (Fig. 2b, c) to meet tilted or in-plane orientation of the far-field director $\mathbf{n}_{0}$ while minimizing the total free energy. However, the topologically protected solitonic structure withstands this dramatic realignment of the $\mathbf{n}_{0}$-background by up to $\approx 90^{\circ}$. The initial axially symmetric solitonic configuration (Fig. 2a) morphs to become asymmetric with respect to a vertical plane parallel to the new in-plane orientation of electrically rotated $\mathbf{n}_{0}$ (Fig. $2 \mathrm{c}, \mathrm{f}$ ). To gain insights into this field-driven transformation of baby skyrmions, we use numerical modeling of their equilibrium structures by minimizing the total free energy $W$ of the chiral nematic LC via the relaxation 


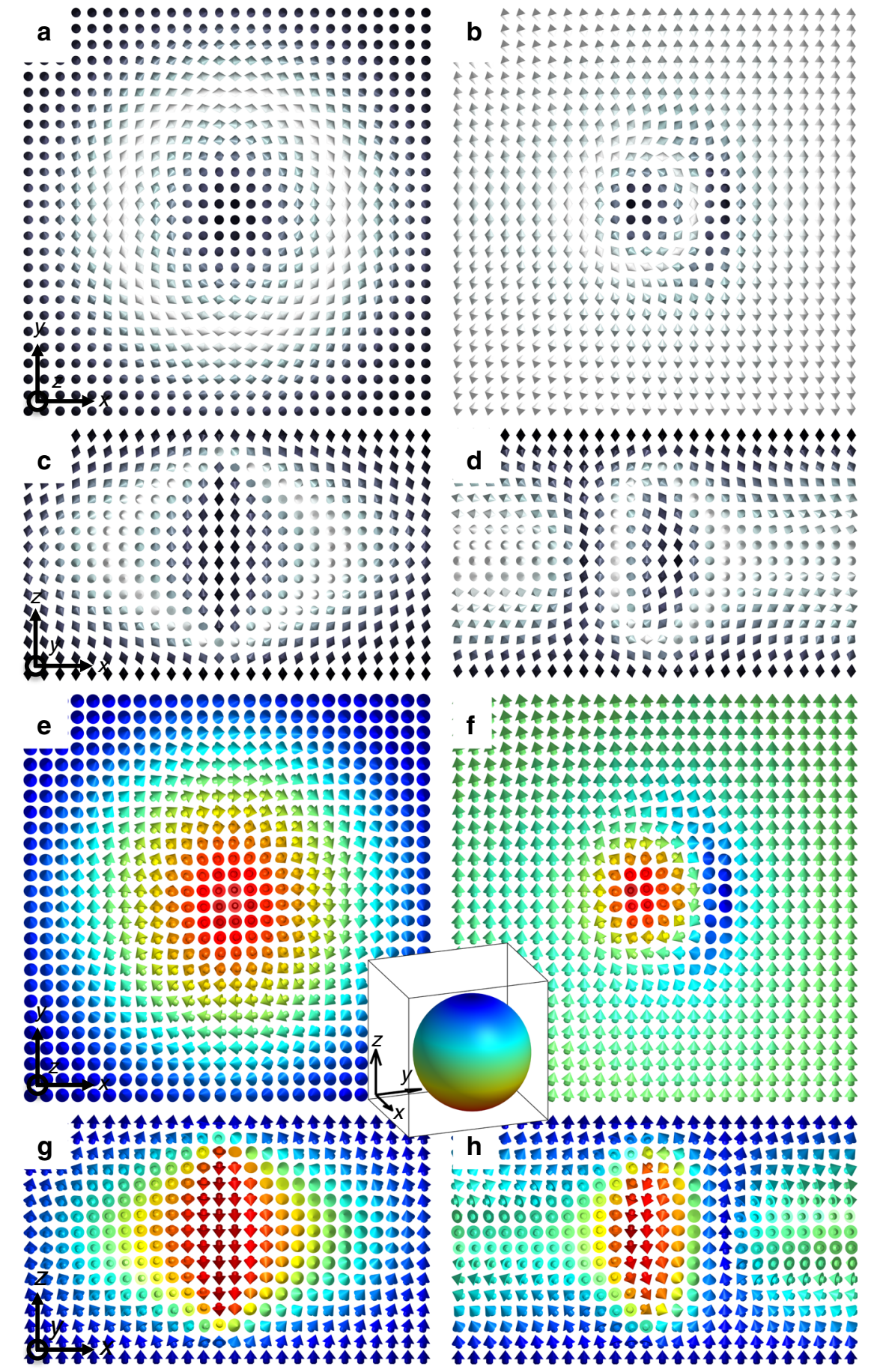

Fig. 3 Three-dimensional structure of baby skyrmions in a confined LC. $\mathbf{a}, \mathbf{b}$ Director structure of the skyrmion in the cross-sectional plane orthogonal to $\mathbf{n}_{0}$ for (a) $U=0 \mathrm{~V}$ and (b) $U=4 \mathrm{~V}$ presented using double cones locally aligned with their axes along $\mathbf{n}(\mathbf{r})$. c, $\mathbf{d}$ The corresponding director structure of the skyrmion in the cross-sectional plane parallel to $\mathbf{n}_{0}$ and passing through the skyrmion's center for (c) $U=0 \mathrm{~V}$ and (d) $U=4 \mathrm{~V}$. e-h The corresponding director structure of the skyrmion in the $(\mathbf{e}, \mathbf{f})$ in-plane and $(\mathbf{g}, \mathbf{h})$ vertical cross-sections visualized using arrows colored according to the corresponding points on $S^{2}$ (inset) and to the coloring scheme also used in Fig. 2

$\operatorname{method}^{14,21}$ :

$$
\begin{gathered}
W=\int\left\{\frac{K_{11}}{2}(\nabla \cdot \mathbf{n})^{2}+\frac{K_{22}}{2}\left[\mathbf{n} \cdot(\nabla \times \mathbf{n})+q_{0}\right]^{2}\right. \\
\left.+\frac{K_{33}}{2}[\mathbf{n} \times(\nabla \times \mathbf{n})]^{2}-\frac{\varepsilon_{0} \Delta \varepsilon}{2}(\mathbf{E} \cdot \mathbf{n})^{2}\right\} \mathrm{d} V
\end{gathered}
$$

where $q_{0}=2 \pi / p$ is the equilibrium chiral wavenumber, $K_{11}, K_{22}$ and $K_{33}$ are Frank elastic constants that pertain to splay, twist and bend distortions of $\mathbf{n}(\mathbf{r})$, respectively, $\Delta \varepsilon$ is the dielectric anisotropy (Table 1) and integration is over the sample's volume. Since the boundary conditions at the confining substrates used in experiments are strong and always satisfied ${ }^{14}, W$ contains only the bulk terms of free energy and the surface energy does not enter this minimization problem (we note that the baby 

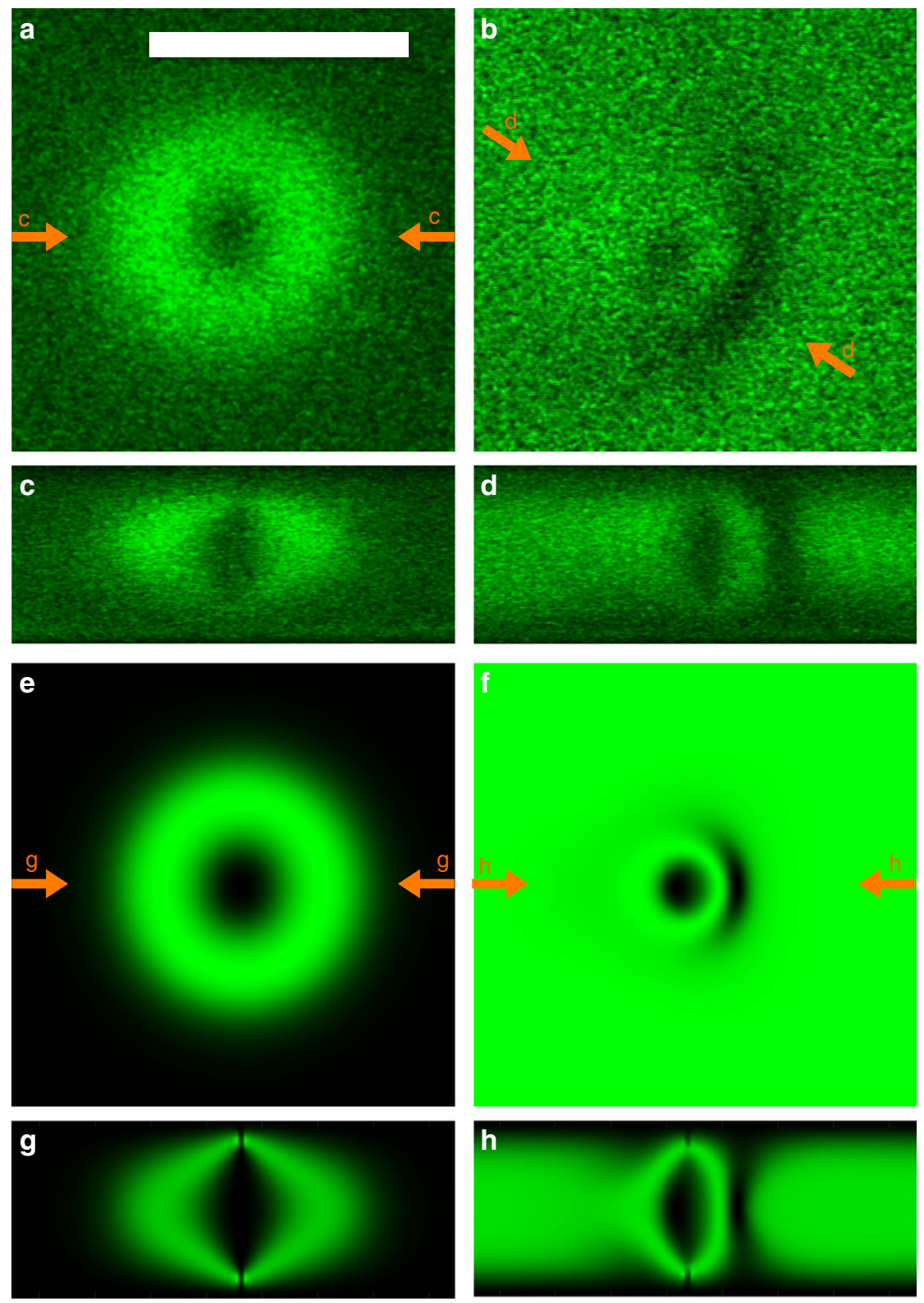

Fig. 4 3PEF-PM images of baby skyrmions. $\mathbf{a}$, $\mathbf{b}$ Experimental 3PEF-PM images of the director structure of the skyrmion in the cross-sectional plane orthogonal to the skyrmion's axis and parallel to substrates for $(\mathbf{a}) U=0 \mathrm{~V}$ and $(\mathbf{b}) U=4 \mathrm{~V}$. $f_{\mathrm{c}}=1 \mathrm{kHz}$. The scale bar in a is $10 \mu \mathrm{m}$ and applies to images (ad). c, d The corresponding experimental 3PEF-PM images of the director structure of the-skyrmion obtained in the vertical cross-sectional plane parallel to the normal to cell substrates and passing through the skyrmion's center for (c) $U=0 \mathrm{~V}$ and (d) $U=4 \mathrm{~V}$. e-h The corresponding computer simulated $3 \mathrm{PEF}$ PM images of the skyrmion in the $(\mathbf{e}, \mathbf{f})$ plane perpendicular to $\mathbf{n}_{0}$ and $(\mathbf{g}, \mathbf{h})$ vertical cross-sections parallel to $\mathbf{n}_{0}$. The 3 PEF-PM excitation light used in imaging was circularly polarized

skyrmions can be also realized in cells with weak perpendicular boundary conditions ${ }^{15}$, but this type of cell confinement is outside the scope of the present study). The energy-minimizing configurations of baby skyrmions (Fig. 2d-f), which emerge from a competition of LC elasticity and the dielectric coupling of $\mathbf{n}$ with electric field $\mathbf{E}$, are consistent with the POM micrographs (Fig. 2a-c).

Figure 3 shows the nonpolar (Fig. 3a-d) and vectorized (Fig. $3 \mathrm{e}-\mathrm{h}$ ) representations of numerically simulated equilibrium director structures of the studied solitons before and after applying the electric field. These computer-simulated director structures are consistent with the experimental reconstruction of the director field. To demonstrate this, Fig. 4 compares experimental and computer-simulated three-photon excitation fluorescence polarizing microscopy (3PEF-PM) images corresponding to the director structures depicted in Fig. 3 for the case of circular polarization of the 3PEF-PM excitation light ${ }^{18}$. The good agreement between the experiment and modeling supports our interpretation of the experimental findings. Furthermore, a similar good agreement between experimental (Fig. 2g, h) and computer-simulated (Supplementary Fig. 1) structures is found also for the baby skyrmion oriented with its axis orthogonal to $\mathbf{n}_{0}$ and the translationally invariant $2 \mathrm{D}$ plane containing $\mathbf{n}_{0}$.

Topology of baby skyrmions in chiral LCs. The baby skyrmion $\mathbf{n}(\mathbf{r})$-structures that we study belong to a diverse family of solitons called 'torons', which contain localized twisted domains 


\begin{tabular}{|llll|}
\hline \multicolumn{2}{l}{ Table 1 Material parameters } & & \\
Material/parameters & MLC-6609 & ZLI-2806 & $\mathbf{5 C B}$ \\
\hline$\varepsilon_{\perp}$ & 7.1 & 8.1 & 5.2 \\
$\varepsilon_{\|}$ & 3.4 & 3.3 & 19 \\
$\Delta \varepsilon$ & -3.7 & -4.8 & 13.8 \\
$K_{11}(\mathrm{pN})$ & 17.2 & 14.9 & 6.4 \\
$K_{22}(\mathrm{pN})$ & 7.51 & 7.9 & 3 \\
$K_{33}(\mathrm{pN})$ & 17.9 & 15.4 & 10 \\
$\gamma(\mathrm{mPas})$ & 162 & 240 & 77 \\
$N_{\mathrm{e}}$ & 1.5514 & 1.518 & 1.726 \\
$N_{\circ}$ & 1.4737 & 1.474 & 1.533 \\
$\Delta N$ & 0.0777 & 0.044 & 0.193 \\
\hline
\end{tabular}

Parameters of the chiral nematic LCs used in our studies, including dielectric $\varepsilon_{\perp}$ and $\varepsilon_{\|}$and elastic $K_{11}, K_{22}$ and $K_{33}$ constants, rotational viscosity $\gamma$, extraordinary and ordinary refractive indices $N_{e}$ and $N_{o}$ and both optical $\Delta N$ and dielectric $\Delta \varepsilon$ anisotropy values. For, comparison, in addition to the parameters of MLC-6609, we also present parameters of two other nematic hosts for which we also observed dynamics of skyrmionic structures, which will be reported elsewhere. The physical parameters presented in the table were measured at $20^{\circ} \mathrm{C}$. The dielectric constant values are reported for the $1 \mathrm{kHz}$ field and the refractive indices are provided for a monochromatic light at $589.3 \mathrm{~nm}$ wavelength

embedded in a uniform far-field background with the help of point or ring-like defects ${ }^{14}, 15,21,22$. Various torons can occur spontaneously within the so-called 'cholesteric bubble' textures ${ }^{15}$ and can be controllably generated by laser tweezers ${ }^{14}$. The twisted regions of $\mathbf{n}(\mathbf{r})$ within torons take the form of $\pi$-twist skyrmion tubes parallel to $\mathbf{n}_{0}{ }^{15,21}$, or various closed rings of half-skyrmions $\pi / 2$-twist and skyrmions with with integer numbers of $\pi$-twist looped around an axis parallel to $\mathbf{n}_{0}{ }^{14,21,22}$. The diverse structures of torons can be classified based on singular point and ring defects (with three distinct species originally identified in ref. ${ }^{14}$ ), by probing the torus knot topology associated with the streamlets tangent to $\mathbf{n}(\mathbf{r})^{14,22}$, and by analyzing both the skyrmion numbers ${ }^{15,21}$ and linking of closed-loop preimages within the twisted regions $^{21}$, as we recently summarized elsewhere ${ }^{21}$. In the present work, we focus on the simplest elementary type of torons, though future studies can potentially extend the exploration of similar types of dynamics to more complex toron and hopfion structures $^{21}$.

At no fields, the baby skyrmion within the elementary toron structure features a $\pi$-twist of $\mathbf{n}(\mathbf{r})$ from its center to periphery in all radial directions, smoothly embedded in the uniform $\mathbf{n}_{0}$ background (Fig. 2a, d). Characterization of topology and energetic stability of these static localized field configurations was a subject of many recent studies ${ }^{14}, 15,21,23-26$, so we discuss here only the topological aspects essential to understanding the electrically-driven transformation and dynamics of $\mathbf{n}(\mathbf{r})$ structures that we study in this work. The director has nonpolar symmetry, $\mathbf{n} \equiv-\mathbf{n}$, but it is common to vectorize $i^{27,28}$ (decorate with a vector $\mathbf{n}$ ) to analyze the topology of field configurations and defects (Fig. 2). We therefore decorate $\mathbf{n}(\mathbf{r})$ and $\mathbf{n}_{0}$ with vectors $^{27,28}$. Mapping the vectorized $\mathbf{n}(\mathbf{r})$ from the sample's midplane to the corresponding order parameter space, the twosphere $S^{2}$ describing all possible orientations of a unit vector $\mathbf{n}(\mathbf{r})$, covers the $S^{2}$ once (Fig. 2a, d), consistent with the topologically protected nature of an elementary baby skyrmions ${ }^{10}$. This topology of the solitons remains unchanged at all used voltages (Fig. 2a-f) and is characterized by the same skyrmion number, an element of the second homotopy group $\pi_{2}\left(S^{2}\right)=Z$ for vectorized and $\pi_{2}\left(S^{2} / Z_{2}\right)=Z$ for unvectorized $\mathbf{n}(\mathbf{r})$, despite the dramatic field-driven transformation of the localized solitonic structures. Because of the actual nonpolar nature of the LC director, $\mathbf{n}(\mathbf{r}) \equiv-\mathbf{n}(\mathbf{r})$ and, similar to the case of point defects ${ }^{28}$, each elementary soliton that we study is characterized by a skyrmion number $\pm 1^{14,15}, 21$, with the sign dependent only on the chosen vectorization direction. The most widely known skyrmions have axisymmetric translationally invariant (along their axes) 2D field configurations, like the one shown in Fig. 2d. However, the intense recent studies of skyrmionic structures in chiral LCs and ferromagnets revealed also non-axisymmetric skyrmions ${ }^{29}$ and skyrmions with the effective cross-section shrinking or expanding along their length in response to boundary conditions, fields and other factors $14,15,22-26,29-32$, and even skyrmions terminating, branching or nucleating at point defects ${ }^{22,32}$. Topological solitons with larger skyrmion numbers and also with nonzero Hopf indices can be realized in confined chiral nematic LCs as well $^{15,21}$, but here we focus on the structures characterized by zero Hopf index and elementary skyrmion numbers \pm 1 . The baby skyrmion in our case is matched to the perpendicular surface boundary conditions of the LC cell by two hyperbolic point defects of opposite hedgehog charges occurring near the confining surfaces ${ }^{14,22}$. The relative charges of point defects were determined according to the recently introduced procedure of analyzing $\mathbf{n}(\mathbf{r})$-textures with multiple point singularities ${ }^{28}$, which we have applied to toron structures as described elsewhere $^{21,22}$. One can present the studied soliton as a baby skyrmion terminating near the cell substrates, with the termination points corresponding to the point defects (Fig. $3 \mathrm{c}, \mathrm{d}, \mathrm{g}, \mathrm{h})^{21}$, 32. This observation is natural as the $\pi_{2}\left(S^{2}\right)=\mathrm{Z}$ and $\pi_{2}\left(S^{2} / \mathrm{Z}_{2}\right)=\mathrm{Z}$ skyrmions are topologically distinct from the uniform field defined along $\mathbf{n}_{0}$ by the surface boundary conditions and, in order to meet these boundary conditions, have to be terminated at their two ends by point defects of the corresponding opposite $\pi_{2}\left(S^{2}\right)=\mathrm{Z}$ and $\pi_{2}\left(S^{2} / Z_{2}\right)=\mathrm{Z}$ hedgehog charge ${ }^{21}, 32$, which in our case is \pm 1 .

The applied electric field $\mathbf{E}$ morphs the director structure but retains its topology (Fig. $3 \mathrm{~b}, \mathrm{~d}, \mathrm{f}, \mathrm{h}$ ), including the skyrmion number of the topological soliton and the hedgehog charges of point defects near the confining surfaces. Despite different structural details, baby skyrmions with the two different orientations (having the $2 \mathrm{D}$ planes parallel and orthogonal to the confining cell substrate normals and $\mathbf{n}_{0}$ at no applied fields) are characterized by the same skyrmion number \pm 1 . We confirm this by mapping the vectorized director field from both the experimental and computer-simulated $2 \mathrm{D}$ cross-sections of these structures orthogonal to the axes of skyrmions to $S^{2}$ (Fig. 2d-f). Smooth changes of $\mathbf{n}_{0}$ in response to $\mathbf{E}$ do not alter topology of such elementary skyrmionic structures but continuously rotate $\mathbf{n}_{0}$ relative to the $2 \mathrm{D}$ plane of the baby skyrmion and the LC cell's midplane from orthogonal (Figs. 1a, 2a, d) to parallel (Figs. 1b, 2c, f). Such two mutual orientations of $\mathbf{n}_{0}$ and the skyrmion's $2 \mathrm{D}$ plane, orthogonal (Fig. 2a, d) and parallel (Fig. 2g-i), are also observed in our experiments and modeling at no fields (Fig. 2g-i and Supplementary Fig. 1), as well as have been previously observed in solid-state ferromagnets ${ }^{31}$. We track positions of spatial regions with vectorized $\mathbf{n}(\mathbf{r})$ corresponding to the north and south poles of $S^{2}$ (dubbed 'preimages' of these $S^{2}$-points) vs. voltage on the basis of POM video microscopy. This yields a linear dependence of the separation between the preimages of the north- and south-poles of $S^{2}$ vs. voltage, with a good agreement between experimental and simulated dependencies and POM images (Fig. 5a, b).

Brownian motion of baby skyrmions. Baby skyrmions undergo Brownian motion, which resembles that of colloidal particles (Fig. 5c, d), consistent with their particle-like nature. Lateral diffusion of the skyrmions is driven by unbalanced orientational thermal fluctuations of $\mathbf{n}(\mathbf{r})$ within their localized structure ${ }^{1}$ and is resisted by the LC's orientational viscous drag associated with the rotational viscosity $\gamma^{1}$. Interestingly, such diffusion is voltagedependent (Fig. 5d) when probed along the $y$-axis but not along 

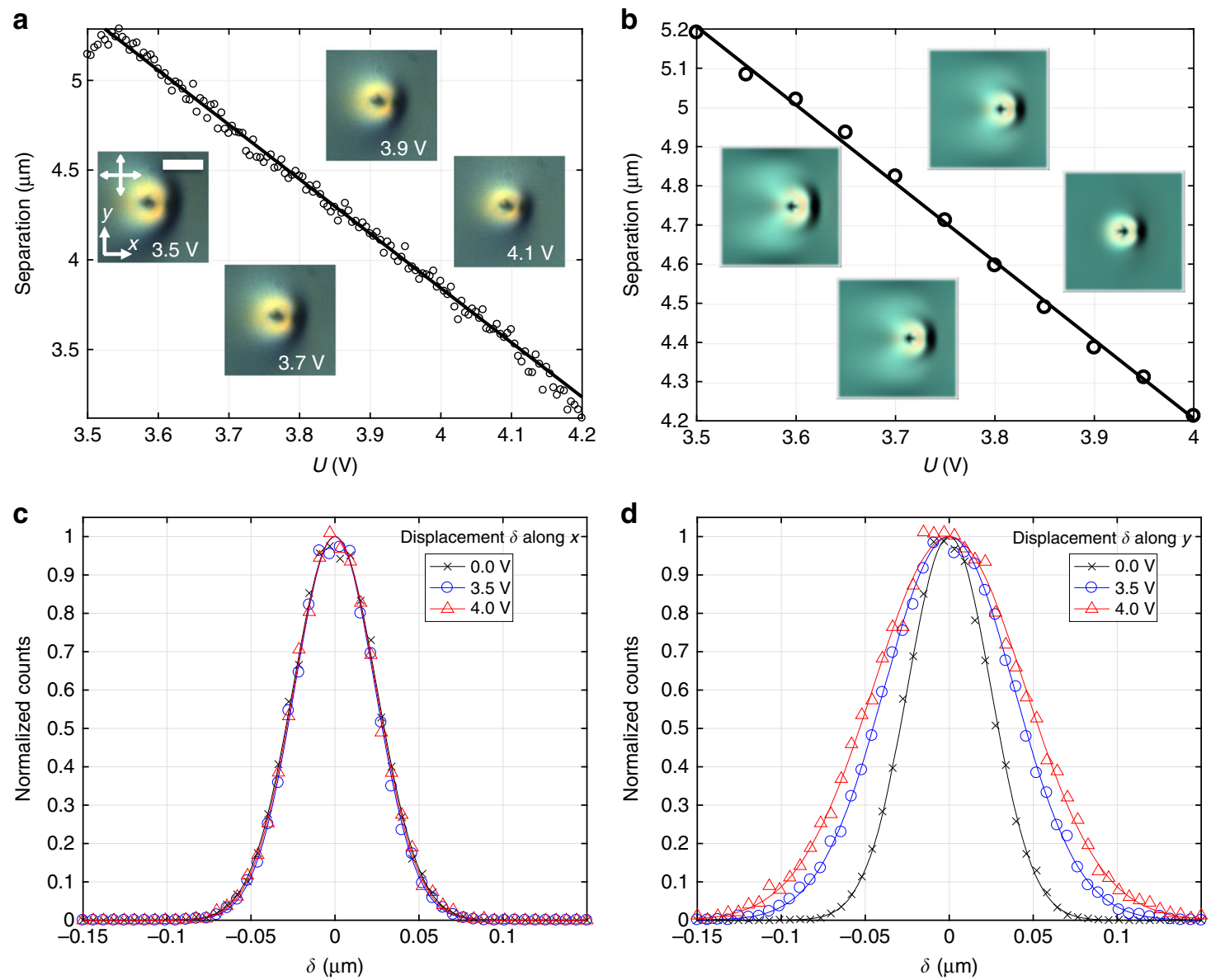

Fig. 5 Electric control and Brownian motion of baby skyrmions. a, b Lateral separation between north-pole (blue arrows in Fig. 2d-f) and south-pole (red arrows) preimages characterized (a) using POM videos and (b) computer simulations. Insets show experimental and computer-simulated POM micrographs at voltages and for crossed polarizers (white double arrows) marked in a. The scale bar in the insets is $10 \mu \mathrm{m}$. $f_{\mathrm{c}}=1 \mathrm{kHz}$. c, $\mathbf{d} \mathrm{Histograms}$ of displacement $\delta$ of the south-pole preimage characterizing Brownian motion of a baby skyrmions (c) along the $x$ axis and (d) along the $y$ axis at different $U$; the solid lines are Gaussian fits of the experimental data points (symbols)

\section{Table 2 Lateral diffusivity $D$ and effective viscous drag coefficients $\zeta$ of skyrmions}

\begin{tabular}{|c|c|c|c|c|}
\hline \multirow[b]{2}{*}{$\underline{U}(\mathrm{~V})$} & \multicolumn{2}{|l|}{ Along $x$} & \multicolumn{2}{|l|}{ Along $y$} \\
\hline & $D\left(\mu m^{2} s^{-1}\right)$ & $\zeta\left(\mathrm{kg} \mathrm{s}^{-1}\right)$ & $D\left(\mu m^{2} s^{-1}\right)$ & $\zeta\left(\mathrm{kg} \mathrm{s}^{-1}\right)$ \\
\hline 0.0 & 0.0187 & $2.174 \times 10^{-7}$ & 0.0185 & $2.195 \times 10^{-7}$ \\
\hline 3.5 & 0.0172 & $2.356 \times 10^{-7}$ & 0.0447 & $9.081 \times 10^{-8}$ \\
\hline 4.0 & 0.0180 & $2.259 \times 10^{-7}$ & 0.0616 & $6.590 \times 10^{-8}$ \\
\hline
\end{tabular}

the $x$-axis (Fig. $5 \mathrm{c}$ ), where these axes are defined with respect to the direction of tilting of $\mathbf{n}_{0}$ in the cell midplane in response to $\mathbf{E}$, which is due to anisotropic morphing of these localized structures in applied field. By fitting the experimental data with Gaussians, we determine half-widths $\Delta$ of histograms of displacements and calculate both the lateral diffusivity of baby skyrmions $D=\Delta^{2} / \tau$ and the effective viscous drag coefficients according to the Einstein relation $\zeta=k_{\mathrm{B}} T / D$, where $k_{\mathrm{B}}=1.38 \times 10^{-23} \mathrm{~J} \mathrm{~K}^{-1}$ is Boltzmann's constant, $\tau=67 \mathrm{~ms}$ is the elapsed time corresponding to the frame rate of video microscopy in our experiment, and $T$ is the absolute temperature. At no fields, the viscous drag $\zeta \approx 2.3 \times 10^{-7} \mathrm{~kg} \mathrm{~s}^{-1}$ is direction-independent, but it becomes anisotropic and voltage-dependent at $U>2 \mathrm{~V}$, with its values within $\zeta_{\mathrm{x}}=2.2-2.4 \times 10^{-7} \mathrm{~kg} \mathrm{~s}^{-1}$ for $x$ directions and $\zeta_{\mathrm{y}}=0.7-2.2 \times 10^{-7} \mathrm{~kg} \mathrm{~s}^{-1}$ for $y$ directions (Table 2).
Electrically driven directional motion of the skyrmions. Interesting dynamics emerge in response to changes of applied fields. The skyrmion drifts away from its original position along the $x$ direction when the voltage is switched on and drifts in the opposite $-x$ direction (more quickly, but to a smaller overall displacement) when the voltage is turned off (Fig. 6a), where the $x, y$ coordinate axes are once again defined with respect to the direction of tilting of $\mathbf{n}_{0}$ in the cell midplane in response to $\mathbf{E}$ (Fig. 1). A net result of turning voltage on and off is that the baby skyrmion translates laterally because its fore-aft displacement in response to the electric switching is asymmetric with respect to the initial position. The effective Reynolds number ${ }^{1}$ associated with this highly overdamped particle-like motion can be estimated as $\operatorname{Re}=\rho v d / \gamma \approx 10^{-8} \ll 1$, which is based on $d=10^{-5} \mathrm{~m}$, velocity $v=1.5 \times 10^{-7} \mathrm{~m} \cdot \mathrm{s}^{-1}$, LC density $\rho=1200 \mathrm{~kg} \cdot \mathrm{m}^{-3}$ and the rotational viscosity $\gamma=0.16 \mathrm{~Pa} \cdot \mathrm{s} 1,21,33,34$. The in-plane force inducing the skyrmion motion (Fig. 6b), which emerges from the combination of LC elasticity and dielectric response, is balanced by the viscous drag force $F_{\mathrm{d}}=-\zeta \mathrm{d} r / \mathrm{d} t$, similar to that of Brownian motion emerging from rotational thermal fluctuations of $\mathbf{n}(\mathbf{r}, t)$ within the soliton (Fig. $5 c, d)$. Using the drag coefficients $\zeta$ determined from characterizing Brownian motion as described above (Table 2), we find that the maximum in-plane forces $F \approx-F_{\mathrm{d}}$ generated by electric pulses (balanced by the viscous drag) are in the pN-range (Fig. 6b). A sequence of electric pulses propels a skyrmion in a well-defined direction and can be exploited 
a

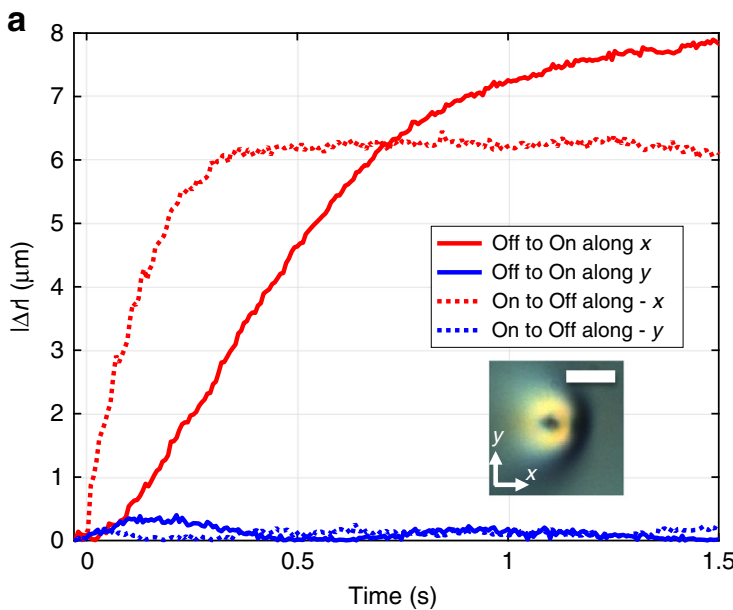

C

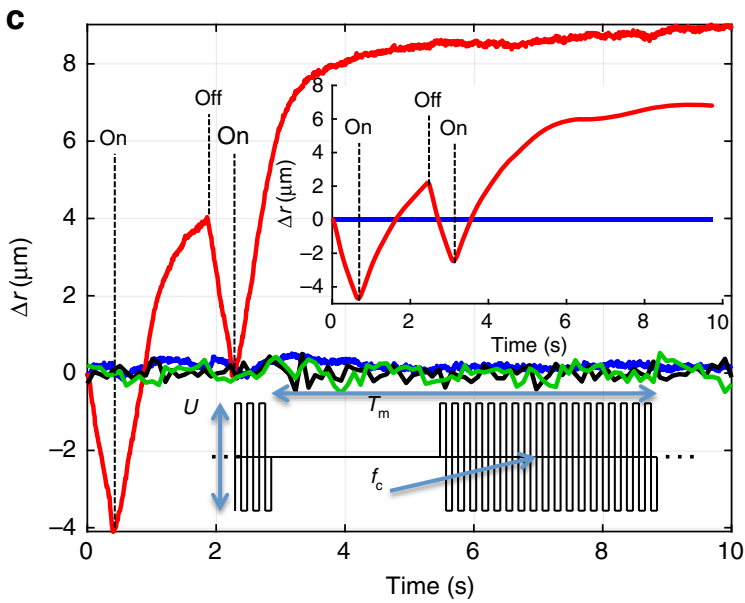

b

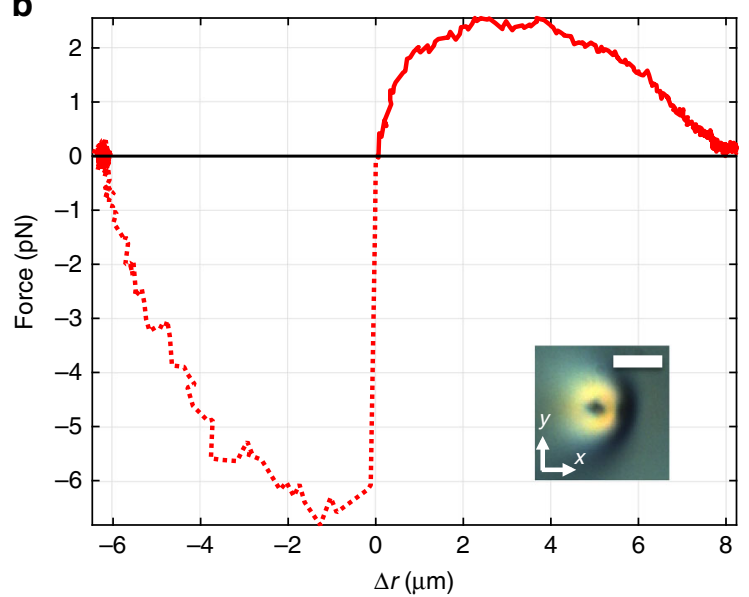

d

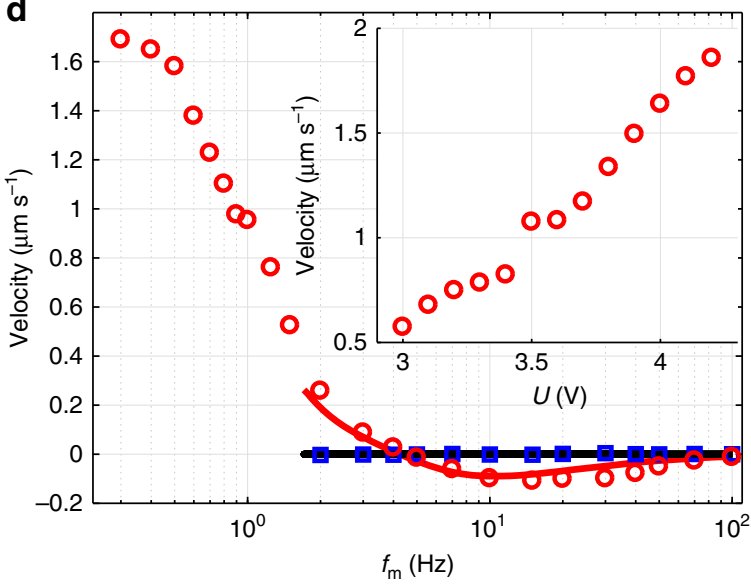

Fig. 6 Directional motion of baby skyrmions. a Relative shift of spatial position of the skyrmion's south-pole preimage $|\Delta r|$ vs. time upon turning voltage $U=4 \mathrm{~V}$ on (solid lines, motion along positive $x, y$ ) and off (dotted lines, motion along negative $x, y$ ) for the $x$ - and $y$-directions defined in the inset and in Fig. $1 \mathrm{c}$ and $2 \mathrm{f}$. (b) Corresponding forces along $\pm x$ (inset) acting on the baby skyrmion vs. $\Delta r$. POM micrographs in the insets of (a, b) define the coordinate system; the scale bar in the insets is $10 \mu \mathrm{m}$. c Video microscopy characterization of the directional motion of the skyrmion in response to switching of the applied voltage on and off when starting with the voltage on; the corresponding computer simulated results are shown in the top inset. The bottom inset illustrates the square waveform voltage driving with carrier frequency $f_{\mathrm{c}}=1 \mathrm{kHz}$ and modulation period $T_{\mathrm{m}}$. The directional motion of the skyrmion along the $x$ axis is compared to the motion of a tracer nanoparticle along the same $x$-axis at no field (black solid line) and at an applied voltage of $4 \mathrm{~V}$ (green solid line) with the same voltage-driving scheme and other conditions. $\mathbf{d}$ Dependence of the direction and amplitude of the baby skyrmion's velocity on the modulation frequency $f_{m}=1 / T_{m}=0.3-100 \mathrm{~Hz}$ characterized using video microscopy for a duty cycle of $75 \%$; the experimental data are shown using symbols and the corresponding computer-simulated dependencies within $f_{m}=1 / T_{m}=2-100 \mathrm{~Hz}$ are shown using solid lines. Inset shows the skyrmion's velocity vs. voltage

for navigating it throughout the LC sample. As an example, skyrmion displacements in response to a few cycles of turning voltage on and off are shown in Fig. 6c. By modulating the applied voltage, we induce the net skyrmion motion in a direction along a vector connecting skyrmion's south- and north-pole preimages (along positive $x$ ) for the relatively large modulation periods $T_{\mathrm{m}}$ (Fig. 6c, bottom inset) and in the opposite $-x$ direction for smaller $T_{\mathrm{m}}$. This behavior is consistent with the difference between the time-dependent $|\Delta r|$ values corresponding to $T_{\mathrm{m}}$ measured in response to turning voltage on and off (Fig. 6a). By varying $f_{\mathrm{m}}=1 / T_{\mathrm{m}}$ within $0.3-100 \mathrm{~Hz}$, the motion direction can be reversed and the skyrmion velocity can be varied from -0.2 to $2 \mu \mathrm{m} \mathrm{s}^{-1}$ (Fig. $6 \mathrm{~d}$ ). The skyrmion moves along $x$ for $f_{\mathrm{m}}<5 \mathrm{~Hz}$ and along $-x$ for $f_{\mathrm{m}}>5 \mathrm{~Hz}$ (Figs. 1c, 6d).

One might suspect that the observed voltage-controlled directional motion of skyrmions could involve the actual LC fluid flows and could be related to hydrodynamic effects, electroconvection, backflows or related phenomena. However, by using high $f_{\mathrm{c}}=1-10 \mathrm{kHz}$ carrier frequencies, we eliminate the possible role that could be played by ionic currents and associated hydrodynamic effects. Moreover, by using plasmonic tracer nanoparticles directly observed using dark field videomicroscopy, we confirm that the observed motion cannot be associated with the LC fluid flows, such as backflows ${ }^{1,33}$ (Fig. 6c). Using holographic laser tweezers, we position the plasmonic nanoparticles at different locations across the sample depth and in proximity or far away from the skyrmions. For the voltagedriving scheme and applied voltages used in our study, tracer nanoparticles exhibit no well-defined directional motion (Fig. 6c), though the backflow effects at $4 \mathrm{~V}$ cause barely noticeable shifting of nanoparticles back and forth as voltage is modulated, with the maximum observed effect shown in Fig. 6c while being compared to the corresponding lateral translations of skyrmions. Even though these weak backflows are detectable (Fig. 6c) and result in self-compensating back-forth translations of nanoparticles somewhat stronger than the Brownian motion of the same nanoparticles, they cannot account for the origin of the field-driven dynamics of skyrmions (the direction of these weak backflows 


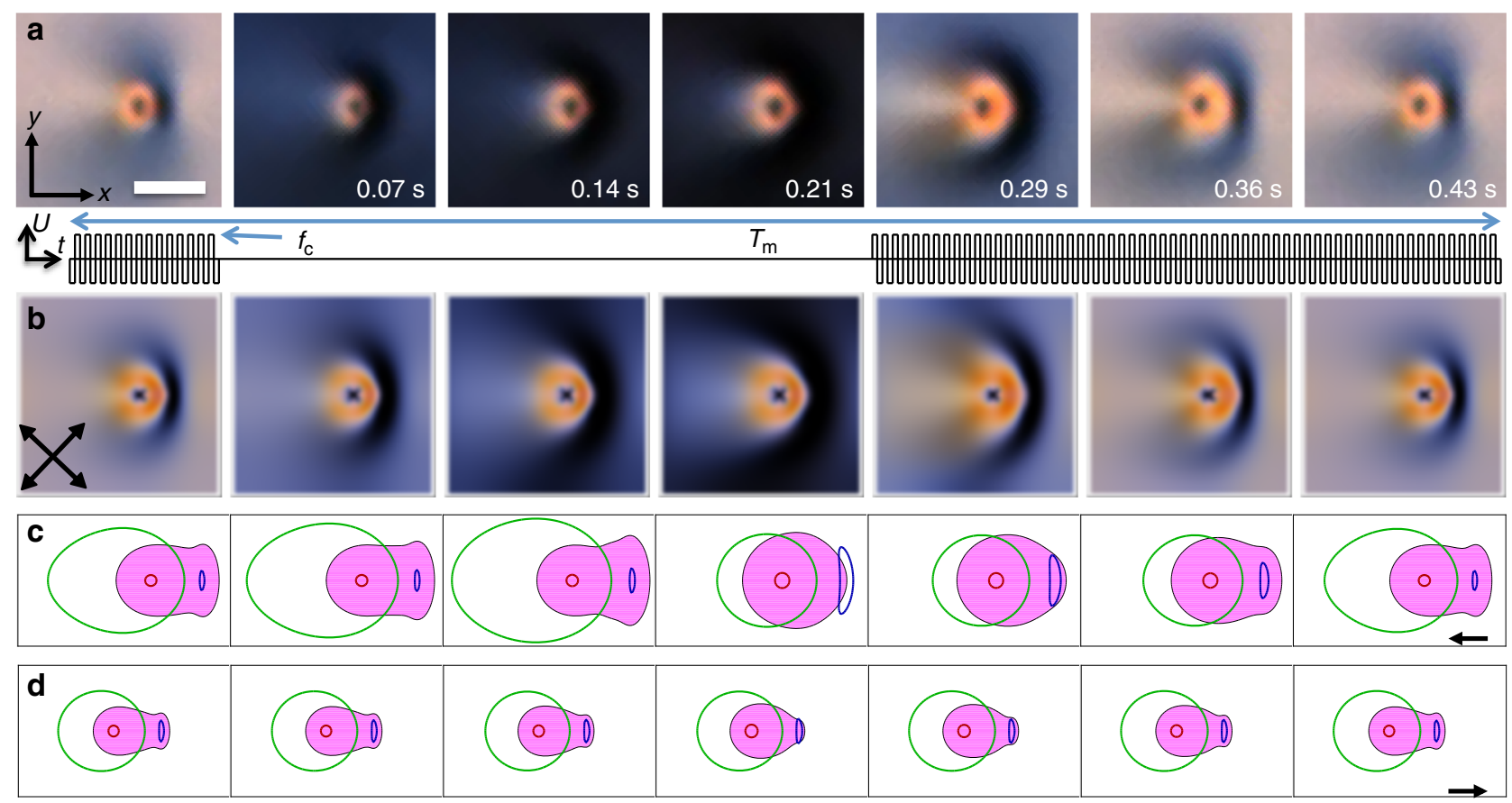

Fig. 7 POM video microscopy and modeling of squirming skyrmions. a Experimental and $\mathbf{b}$ computer-simulated POM micrographs of a baby skyrmion when moving along a vector connecting the south- and north-pole preimages (along positive $x$ ), with the corresponding visualizations of squirming waves in the twisted quasi-particle illustrated in $\mathbf{c}$. The scale bar in the left-side image in $\mathbf{a}$ is $10 \mu \mathrm{m}$ and applies to all POM image frames. The schematic in the inset between experimental and computer-simulated micrographs shows the timing of turning the high-frequency voltage on and off within the elapsed time $t$ corresponding to one modulation period $T_{\mathrm{m}}$, correlated with the micrographs in $\mathbf{a}, \mathbf{b}$. Magenta filled surfaces in $\mathbf{c}$ depict the twisted regions of where the inplane component of $\mathcal{H} / q_{0} \geq 0.1$ of the chiral nematic LC. Crossed polarizers (black double arrows) in $\mathbf{b}$ correspond to both computer-simulated and experimental micrographs. A skyrmion is depicted for frames equally spaced in time during one cycle at $f_{\mathrm{c}}=1 \mathrm{kHz}$ and $f_{\mathrm{m}}=2 \mathrm{~Hz}$. After turning voltage off, the north-pole preimage expands and the south-pole preimage moves away from the north-pole preimage. Once the voltage is reinstated, the north-pole preimage localizes at a larger distance away from the south-pole preimage, and then the north- and south-pole preimages come back together within the laterally compact skyrmion that overall shifts along $x$, exhibiting directional motion. $\mathbf{d}$ Visualization of dynamics of a skyrmion quasiparticle similar to that shown in $\mathbf{c}$ but for $f_{m}=20 \mathrm{~Hz}$ yielding an opposite direction of motion, where magenta filled surfaces depict the twisted regions with $\mathcal{H} / q_{0} \geq 0.05$. In $\mathbf{c}$, $\mathbf{d}$, the red and blue contours enclose the spatial regions of the south- and north-pole preimages and the green contours show the cumulative preimage of the equatorial points of $\mathrm{S}^{2}$. The weaker squirming changes of these preimages and the high- $\mathcal{H}$ region during $T_{\mathrm{m}}$ at $f_{\mathrm{m}}=20 \mathrm{~Hz}$ as compared to $f_{\mathrm{m}}=2 \mathrm{~Hz}$ are consistent with the corresponding values of the absolute velocity (Fig. $5 \mathrm{~d}$ ). Black arrows in $\mathbf{c}, \mathbf{d}$ show the skyrmion's motion directions at corresponding $f_{\mathrm{m}}$

changes across the cell thickness, so that they roughly selfcompensate and do not significantly alter dynamics of the skyrmion motion). Although our tracer nanoparticles interact with the solitons, similar to the case of our previous studies ${ }^{35}$, these interactions do not reveal fluid flows in the skyrmion vicinity, additionally confirming that electroconvection and hydrodynamics effects cannot be responsible for the skyrmion motion. These findings pose a challenge of unraveling the physical origins of the observed soliton dynamics.

To gain insights to the physics behind the directional skyrmion motion, we again resort to numerical modeling. As we modulate the voltage applied to our samples, a viscous torque associated with rotational viscosity $\gamma$ opposes the fast rotation of $\mathbf{n}$ in LCs in response to the competing electric and elastic torques ${ }^{1}$. The resulting director dynamics is governed by a torque balance equation ${ }^{1}, \gamma \partial n_{i} / \partial t=-\delta W / \delta n_{i}$, from which we obtain the temporal evolution $n_{i}(t)$ towards the equilibrium. As a result of the periodic modulation of voltage $U, \mathbf{n}(\mathbf{r}, t)$ evolves in such a way that the computer simulated baby skyrmion translates, similar to what we observe experimentally (Figs. 6, 7), albeit modeling at $f_{\mathrm{m}}<2 \mathrm{~Hz}$ is challenged by the large separation of north- and south-pole preimages during voltage modulation, which make computational calculations very costly. Our modeling reproduces the fine features of the jerky motion of a baby skyrmion (top inset of Fig. 6c), the modulation-frequency dependence of its velocity (inset of Fig. 6d), and even the temporal evolution of experimental POM textures (Fig. 7a, b). In the sample midplane, the non-reciprocal squirming evolution of $\mathbf{n}(\mathbf{r}, t)$ of the soliton within a period $T_{\mathrm{m}}$ of modulating voltage at frequencies $f_{\mathrm{m}}=2 \mathrm{~Hz}$ (Fig. 7c) and $f_{\mathrm{m}}=20 \mathrm{~Hz}$ (Fig. 7d) is consistent with the opposite motion directions (Fig. 6c, d). To show this, we illustrate the evolution of preimages of the north-pole, south-pole, and equatorial regions of $S^{2}$, as well as the in-plane $2 \mathrm{D}$ handedness ${ }^{21}, 36$ $\mathcal{H}=-\mathbf{n} \cdot(\nabla \times \mathbf{n})$ describing twist of $\mathbf{n}(\mathbf{r})$ within the $2 \mathrm{D}$ plane of a baby skyrmion in the cell midplane in a quantitative way (Fig. 7c, d). Interestingly, the high- $\mathcal{H}$ twisted region (colored in magenta) is morphing within $T_{\mathrm{m}}$ in a way that resembles the traveling-wave surface squirming that biological cells ${ }^{16}$ and biomimetic robots ${ }^{17}$ use to swim in low Reynolds number fluid (Fig. 7c, d). The twisted region is squeezed and relaxed as voltage is applied and turned off within $T_{\mathrm{m}}$, but the combination of chirality and coupling of $\mathbf{n}(\mathbf{r})$ to the modulated high-frequency field (present only within a fraction of $T_{\mathrm{m}}$ ) breaks reciprocity of $\mathbf{n}(\mathbf{r}, t)$, resulting in a directional motion of the soliton. The twisted high- $\mathcal{H}$ region of the baby skyrmion can be thought of as a $2 \mathrm{D}$ quasiparticle. The surface waves in this high- $\mathcal{H}$ twisted quasiparticle resemble the squirming traveling waves ${ }^{16,17}$, though they have very different origin as compared to biological and biomimetic systems. Importantly, this squirming motion involves mainly the rotational dynamics of $\mathbf{n}(\mathbf{r})$ and not the actual LC fluid flow, although strong backflows can be potentially induced at high voltages ${ }^{33}$ and may provide a means of further enriching the 

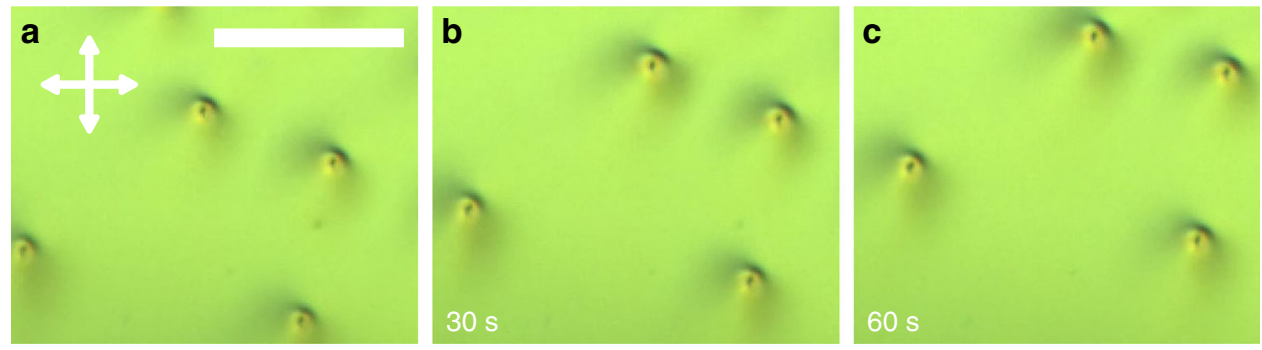

Fig. 8 Collective unidirectional motion of baby skyrmions. a-c POM frames from a POM video. The elapsed time is marked in the bottom-left corners of the images. The orientation of crossed polarizers is shown using white double arrows and the scale bar is $100 \mu \mathrm{m}$

out-of-equilibrium behavior of this solitonic system. For example, at high voltages, the backflow-mediated motion of tracer particles spatially co-located with the skyrmion's localized field configuration may exhibit an interplay with the topology and periodically evolving structure of these topological solitons.

Similar to active colloidal particles ${ }^{2}$, which locally convert chemical energy to self-propel, skyrmions locally convert electric energy due to the modulated applied voltage to produce directional motion by invoking the rotational dynamics of $\mathbf{n}(\mathbf{r}, t)$. The time evolution of solitonic director structures within each modulation period $T_{\mathrm{m}}$, analyzed on the basis of experimental and simulated POM videos (Fig. $7 \mathrm{a}, \mathrm{b}$ ), as well as the high- $\mathcal{H}$ twisted regions and preimages (Fig. $7 \mathrm{c}, \mathrm{d}$ ), is nonreciprocal with respect to turning the high-frequency voltage on and off. The non-reciprocity of $\mathbf{n}(\mathbf{r}, t)$ within $T_{\mathrm{m}}$ of a squirming skyrmion arises mainly from the fact that dielectric and elastic torques drive the director evolution when voltage is turned on but only the elastic torque drives relaxation of the structure upon turning voltage off. Even in the case of LC displays ${ }^{20}$, where switching involves only topologically trivial translationally invariant director structures, this difference in torque balances upon turning voltage on and off causes differences between the rising and falling response times. In the case of baby skyrmions, however, the non-reciprocity of the on- and off-cycles is further enhanced by LC chirality and the fact that the director structure at applied $\mathbf{E}$ is highly asymmetric (Figs. 2e, f, 3, 4), which causes highly asymmetric patterns of dielectric and elastic torque densities. The asymmetry of evolution of $\mathbf{n}(\mathbf{r}, t)$ visually resembles how the low-Reynolds-number motion in biology takes advantage of non-reciprocity of effective and recovery strokes, say due to the beating cycles of cilia ${ }^{16}$. However, one should also understand the limitations of this analogy as compared to active particles and biological cells because, being localized field configurations, our skyrmions have no cell walls, membranes, or physical interfaces. Furthermore, the soliton motion reported here can emerge only in anisotropic media that feature facile response of localized field configurations to external stimuli.

Figure 8 shows a series of frames extracted from a POM video demonstrating that not only individual baby skyrmions exhibit directional motion, but also that multiple skyrmions within the same sample can exhibit such behavior. Interestingly, the dynamics of these different skyrmions is characterized by comparable velocity and the same motion direction (Fig. 8). Furthermore, as the number density of baby skyrmions increases, they start interacting with each other, which further enriches the out-of-equilibrium behavior of these solitonic systems and leads to forms of self-assembly not accessible to the equilibrium structures. Detailed explorations of the non-equilibrium processes of such solitonic systems at large number densities of solitons will be discussed elsewhere.

Diversity of material systems exhibiting skyrmion dynamics. The topological soliton dynamics that we report in this article is not restricted to the particular nematic host material system of MLC-6609, for which we present most of the data, but can be achieved with a broad variety of LC materials with both positive and negative dielectric anisotropy. For example, similar experimental and numerical results are also obtained (to be reported elsewhere) using a chiral nematic LC based on a nematic mixture of ZLI-2806 (see material parameters in Table 1) doped with either a left-handed chiral additive ZLI-811, the same as that used in our current study, or a right-handed additive CB-15 (all materials purchased from Merck). Furthermore, translational motion of baby skyrmions shown in Fig. $2 \mathrm{~g}-\mathrm{i}$ and Supplementary Fig. 1 can be driven electrically even when obtained in materials with positive dielectric anisotropy, such as pentylcyanobiphenyl (5CB, purchased from Frinton Laboratories, Inc.), which we will describe elsewhere. In a material with positive dielectric anisotropy, such as $5 \mathrm{CB}$, the field that is applied perpendicular to substrates squeezes the localized skyrmion structure (such as the one shown in the Supplementary Fig. 1c) asymmetrically. When voltage is modulated (Supplementary Fig. 1c), this too results in motion because of the field-driven asymmetric squirming response of the localized structure.

\section{Discussion}

Several recent studies have highlighted the topological, energetic and other analogies between LC and ferromagnetic solid-state solitons $15,21,23,25,37$. Indeed, within the one-constant approximation $K=K_{11}=K_{22}=K_{33}$ and for the effective exchange constant $A=K / 2$, the Dzyaloshinskii-Moriya constant $D=K q_{0}$ and $\mathbf{n}(\mathbf{r}) \rightarrow \mathbf{m}(\mathbf{r})$, the first three elastic terms of Eq.(1) reduce to become analogous to the simplest description of chiral ferromagnetic solids like MnSi: ${ }^{15}, 21,23,25,37$

$$
W=\int\left\{A(\nabla \mathbf{m})^{2}+D[\mathbf{m} \cdot(\nabla \times \mathbf{m})]\right\} d V
$$

This may provide insights into the physical underpinnings of certain types of dynamics of baby skyrmions in the spin textures and magnetization fields $\mathbf{m}(\mathbf{r})$ of the solid ferromagnetic films, though the coupling of $\mathbf{m}(\mathbf{r})$ to external electric and magnetic fields can be different from the dielectric coupling exploited in this work. By applying the $K=K_{11}=K_{22}=K_{33}$ one-constant approximation in our numerical modeling, we indeed find that the field-driven directional motion of skyrmions persists, though accounting for the LC's actual elastic constants is important for achieving the quantitative agreement between experimental and computer-simulated voltage-dependent structures and motion velocities (Figs. 4-7). Since the solitonic structures can also be realized in the fluid analogs of solid ferromagnetic films that combine properties of optical anisotropy with a facile magnetic response ${ }^{37,38}$, this may enable racetrack memory applications based on both magnetic and electric driving of skyrmion motion and both magnetic and optical data storage. Indeed, our preliminary studies show that periodically modulated magnetic fields 
applied to chiral ferromagnetic LC colloids with the localized skyrmionic structures similar to the ones studied here ${ }^{37,38}$ also induce directional motion that stems from the structural squirming mechanism, similar to the one reported here. Furthermore, since the textures in LCs ${ }^{1}$ and fluid ferromagnets ${ }^{37}$ interact with colloidal microparticles because of the elasticity and boundary conditions on surfaces of particles, their dynamics can be potentially exploited for the delivery of cargo on micrometer scales ${ }^{39}$.

From a fundamental viewpoint, our study is a demonstration of non-annihilating dynamics of $\pi_{2}\left(S^{2}\right)=\mathrm{Z}$ and $\pi_{2}\left(S^{2} / \mathrm{Z}_{2}\right)=\mathrm{Z}$ topological solitons in LCs, very different from previously explored dynamics of both solitons and singular defects in these soft matter systems ${ }^{40-43}$. Experimental and theoretical studies of such non-equilibrium behavior may be also extended to skyrmions with large skyrmion numbers ${ }^{15}$ and to the $\pi_{3}\left(S^{2}\right)=\mathrm{Z}$ and $\pi_{3}\left(S^{2} / Z_{2}\right)=\mathrm{Z}$ topological solitons with linked preimages and nonzero Hopf indices ${ }^{21,37}$. The directional motion of solitons can be also realized in materials with positive dielectric anisotropy and also in response to magnetic fields, light, and other external stimuli. For example, to drive skyrmion motion with magnetic fields one can explore both the diamagnetic response of conventional LCs ${ }^{1}$ (with a quadratic free energy term describing the coupling between $\mathbf{n}$ and magnetic field $\mathbf{B}$ ), or also the ferromagnetic response of chiral LC ferromagnets ${ }^{37}$, in which both the $\pi_{2}\left(S^{2}\right)=\mathrm{Z}$ and $\pi_{3}\left(S^{2}\right)=\mathrm{Z}$ topological solitons have been recently realized $^{37,}, 38$.

At low Reynolds numbers, microswimmers in conventional viscous fluids execute non-reciprocal motions to self-propel ${ }^{20}$. This non-reciprocity requirement is a consequence of the wellknown Purcell's scallop theorem ${ }^{20}$, which also constrains the means of producing directional active motions in $\mathrm{LCs}^{44-47}$. Our findings demonstrate that the non-reciprocal rotational dynamics of LC director yields a mechanism of motion of solitonic particlelike field configurations. This mechanism, characteristic of LC fluids, can be further enriched by other mechanisms already explored in a host of recent studies of conventional fluids and both active LCs and LC colloids, ranging from flagellum-enabled swimming of bacteria ${ }^{44}$ to various electro-kinetic, backflow and hydrodynamic effects associated with asymmetric fluid flows and various instabilities ${ }^{45-47}$. Indeed, we observe that the out-ofequilibrium behavior of baby skyrmions changes when we increase voltage amplitudes to increase the role of backflow effects or reduce the carrier frequency to couple $\mathbf{E}$ with the residual ionic impurities within the LC (to exploit the flows associated with hydrodynamics) ${ }^{1}$. A detailed study of this complex interplay of different motion-enabling mechanisms in LCs is well beyond the scope of our present study and will be reported elsewhere.

To conclude, we have uncovered unexpected directional motion of topological solitons in response to modulated electric fields. This finding bridges the recent key developments in studies of out-of-equilibrium phenomena ${ }^{2-5}, 17,48$ and topological solitons $^{6-15}, 21,37$, and may lead to versatile topology-protected dynamic systems with potential applications in microfluidics, racetrack memory devices and so on. Directional motion of solitons is enabled by non-reciprocal squirming of their particle-like textures and involves mainly the rotational dynamics of the LC director, without relying on the actual fluid flow as a dominant mechanism of motion. These findings establish the particle-like out-of-equilibrium solitonic structures as a kind of stimuli-responsive soft particles. Our observations and numerical analysis may provide insights into the means of inducing directional motion of skyrmionic textures in ferromagnetic solids, with potential impact on the emergent field of skyrmionics ${ }^{12}$.

\section{Methods}

Details of sample preparation and experimental techniques. The LC material for which we report majority of experimental and computational studies is a mixture of a nematic host MLC-6609 and a chiral additive ZLI-811 (both purchased from Merck) with an equilibrium helicoidal pitch $p \approx 10 \mu \mathrm{m}$. This LC mixture was designed to have negative dielectric anisotropy and respond to external electric field in such a way that the director tends to orient orthogonally to the applied field direction. The material parameters of this LC system, including dielectric and elastic constants, rotational viscosity and refractive indices, are provided in Table 1. In addition, test experiments were also preformed for several other material systems (Table 1). In some samples, small number densities (about one particle per $10,000 \mu^{3}$ ) of plasmonic gold nanorods with dimensions about $22 \times 50 \mathrm{~nm}$ were added to the LC for tracing possible fluid flows on the basis of observing the nanoparticle motion using dark field videomicroscopy; the preparation of gold nanorods and their dilute dispersions in LCs is described in details elsewhere ${ }^{49}$.

LC cells were assembled using glass substrates with transparent indium tin oxide (ITO) electrodes treated with polyimide SE1211 (purchased from Nissan) for imposing surface boundary conditions. SE1211 was spin-coated at $2700 \mathrm{rpm}$ for $30 \mathrm{~s}$ and then baked for $5 \mathrm{~min}$ at $90^{\circ} \mathrm{C}$ followed by $1 \mathrm{~h}$ baking at $180^{\circ} \mathrm{C}$ to provide strong vertical surface boundary conditions for the LC director. For some of the studied samples, by weakly rubbing SE1211-coated glass plates with initially perpendicular boundary conditions for $\mathbf{n}$, we have defined a small $\left(1-3^{\circ}\right)$ pretilt away from these perpendicular conditions (see ref. ${ }^{50}$ for more details), so that the direction of tilting of $\mathbf{n}$ during electric switching could be well defined, which was then pre-determining the direction of skyrmion motion as discussed above. The LC cell gap was set to be $10 \mu \mathrm{m}$ using glass-fiber segments dispersed in ultravioletcurable glue. Small drops of glue with the spacers were squeezed between the glass substrates with inward-facing ITO electrodes and alignment layers and the glue was then cured using ultraviolet exposure to obtain LC cells with the desired cell gap. The LC material was infiltrated into the cells by means of capillary forces and then the cell edges were sealed with 5-min fast-setting epoxy. Electric wires were soldered to the ITO electrodes and used to apply voltages. Different voltage driving schemes and waveforms were produced using a homemade LabView-based software and the data acquisition board (NIDAQ-6363, National Instruments) and applied to the LC cells using the wires soldered to the ITO. An amplitude modulated $1-10 \mathrm{kHz}$ high-frequency square wave was used to avoid hydrodynamic instabilities and other types of complex behavior associated with ions at lowfrequency applied fields. The voltage driving-scheme was based on the $f_{\mathrm{c}}=1 \mathrm{kHz}$ or $f_{\mathrm{c}}=10 \mathrm{kHz}$ square-wave carrier frequency modulated by a lower frequency $f_{\mathrm{m}}$ square-wave. The relevant parameters of the voltage driving scheme are illustrated in Fig. 1, in the bottom inset of Figs $6 \mathrm{c}, 7 \mathrm{a}$.

Non-contact generation and manipulation of solitonic configurations was achieved using holographic laser tweezers and a tightly focused $1064 \mathrm{~nm}$ laser at powers of less than $50 \mathrm{~mW}$, as described in detail elsewhere ${ }^{14}, 15$. For this, we utilized an Ytterbium-doped fiber laser (YLR-10-1064, IPG Photonics, operating at $1064 \mathrm{~nm}$ ) and a phase-only spatial light modulator (P512-1064, Boulder Nonlinear Systems) integrated into a holographic laser tweezers setup capable of producing arbitrary $3 \mathrm{D}$ patterns of laser light intensity within the sample ${ }^{14,15}$. The laser tweezers were also integrated with the $3 \mathrm{D}$ nonlinear optical imaging setup described below, enabling fully optical generation, control, and nondestructive imaging of the solitons. The physical mechanism behind the laser generation of solitons is the optical Fréedericksz transition, the realignment of the LC director away from the far-field background $\mathbf{n}_{0}$ caused by its coupling to the opticalfrequency electric field of the laser beam, which is described by a corresponding term of free energy ${ }^{14,15}$. This coupling, enriched by holographically generated patterns of the trapping laser beam's intensity, phase singularities, and translational motion of individual traps ${ }^{14,15}$, prompts complex director distortions that relax to the global or local elastic free energy minima, some of which are the baby skyrmions we study in this work. Similar solitons were also generated by means of applying low-frequency fields and quenching the frustrated homeotropic chiral LC cells from isotropic phase to the mesophase ${ }^{15,21,22}$.

Polarizing optical microscopy observations in the transmission mode were achieved with a multi-modal imaging setup built around the IX-81 Olympus inverted microscope (also includes the nonlinear optical imaging technique described below) and charge coupled device cameras. Temporal evolution of POM textures and motions of colloidal particles were recorded with a camera Flea FMVU-13S2C-CS (purchased from Point Grey Research, Inc.) or a Spot 14.2 Color Mosaic (purchased from Diagnostic Instruments, Inc.) or a fast camera HotShot 512SC (purchased from NAC Image Technology, Inc.). The same high numerical aperture (NA) Olympus objective $(100 \times, \mathrm{NA}=1.4)$ was used for the polarizing optical microscopy, laser manipulation and nonlinear optical imaging described below. Optical imaging additionally utilized $10 \times, 20 \times$, and $50 \times$ dry objectives with numerical aperture NA $=0.3-0.9$. The sequences of video microscopy frames were analyzed using ImageJ software and its plugins (freeware from $\mathrm{NIH}$ ), which allowed for tracking lateral positions of the north-pole and south-pole preimages (which both appear dark in the POM images) within the baby skyrmions with $7-10 \mathrm{~nm}$ resolution and the temporal resolution corresponding to the camera's frame rates, which was 1000 frames per second for the fast camera HotShot 512SC.

Nonlinear optical imaging was performed using three-photon excitation fluorescence polarizing microscopy (3PEF-PM) setup built around a IX-81 
Olympus inverted optical microscope ${ }^{21}$. The polarized self-fluorescence from the LC molecules was detected within the $400-450 \mathrm{~nm}$ spectral range and excited through a process of three-photon absorption using a Ti-Sapphire oscillator (Chameleon Ultra II, Coherent) operating at $870 \mathrm{~nm}$ with $140 \mathrm{fs}$ pulses at a repetition rate of $80 \mathrm{MHz}$. The 3PEF-PM signal was collected through an oilimmersion $100 \times$ objective with numerical aperture of 1.4 and detected by a photomultiplier tube (H5784-20, Hamamatsu). We scanned the excitation beam through the sample volume with the help of galvano-mirrors (in lateral directions) and a stepper motor (across the sample thickness) and recorded the 3PEF-PM signal as a function of coordinates, which was then used to construct 3D images by means of ParaView (freeware obtained from KitwarePublic) ${ }^{21}$. The linear polarization of the excitation beam was controlled using a polarizer and a rotatable half-wave retardation plate. The detection channel utilized no polarizers. The 3PEF-PM intensity scaled as $\propto \cos ^{6} \psi$, where $\psi$ is the angle between $\mathbf{n}(\mathbf{r})$ and the excitation beam's linear polarization ${ }^{18,21}$ (assumed to remain unchanged despite the beam focusing through dielectric interfaces and the weakly birefringent LC medium, with the sample design minimizing these changes) and was used to reconstruct the $\mathbf{n}(\mathbf{r})$ patterns ${ }^{18,21}$. The reconstruction of the $3 \mathrm{D}$ solitonic $\mathbf{n}(\mathbf{r})$-structures took advantage of the self-fluorescence patterns obtained at different polarizations of excitation light, as described elsewhere ${ }^{18,21}$. In order to eliminate the ambiguity between the two possible opposite $\mathbf{n}(\mathbf{r})$ tilts in the analysis of 3D images, additional cross-sectional 3PEF-PM images were obtained at orientations of the LC cell's normal tilted by $\pm 2^{\circ}$ with respect to the microscope axis for linear polarizations of excitation laser light parallel or perpendicular to the plane of the corresponding vertical cross-sectional image. The $\mathbf{n}(\mathbf{r})$ tilt ambiguity was then eliminated based on the $\propto \cos ^{6} \psi$ scaling of the 3PEF-PM signal and the ensuing spatial changes of intensity prompted by the $\pm 2^{\circ}$ tilts. To further narrow the angular sector of $\mathbf{n}$-orientations corresponding to preimages of points on $S^{2}$ with target azimuthal angles $\varphi$, we each time obtained three 3D images with azimuthal orientation of the linear polarization of excitation beam at $\varphi$ and $\varphi \pm 3^{\circ}$. These $3 \mathrm{D}$ images were smoothed using Matlab-based software and then used in a differential analysis to improve orientational resolution of imaging the director field to better than $\pm 3^{\circ}$.

Details of numerical modeling. In computer simulations, three-dimensional grids of $112 \times 112 \times 32$ equally spaced points were used with periodic boundary conditions along lateral edges of the computational volume and perpendicular boundary conditions at the top and bottom planes ${ }^{14,21,51}$. The utilized values of the elastic, dielectric, optical and viscous constants are summarized in the Table 1. To obtain the equilibrium director structures at different applied fields, starting from random initial conditions, $\mathbf{n}(\mathbf{r})$-configurations were relaxed using a centered finite difference scheme to calculate the functional derivatives $\delta W / \delta n_{i}$ of the free energy given by Eq. (1), where $n_{i}$ is the component of $\mathbf{n}$ along the $i^{\text {th }}$ axis $(i=x, y, z)$. The maximum stable time step $\Delta t$ was used to change the components of $\mathbf{n}$ by an elementary displacement calculated as described in detail elsewhere ${ }^{14,21}$. The new $\mathbf{n}$ was normalized after each time step. This process was repeated until the grid averaged elementary displacement was less than $10^{-14}$. Director dynamics was modeled using the torque balance equation, $\gamma \partial n_{i} / \partial t=-\delta W / \delta n_{i}$, from which we obtained the temporal evolution $n_{i}(t)$ towards the equilibrium at a given applied voltage $U$. The computer-simulated director structures of baby skyrmions were then used in modeling of polarizing optical micrographs by means of the Jones-matrix approach ${ }^{15}$ for the experimental material and cell parameters, such as optical anisotropy, equilibrium cholesteric pitch, and cell thickness (Table 1).

The $\mathbf{n}(\mathbf{r})$-configurations in the vertical cross-sections of the solitonic structures were represented in the form of arrays of azimuthal and polar angles. To utilize the Jones-matrix method for modeling of POM textures ${ }^{15}$, we split the cell into a stack of 32 thin sub-layers while assuming that the orientation of $\mathbf{n}(\mathbf{r})$ is constant across the thickness of one of these sub-layers. The corresponding coordinate-dependent Jones matrices had an optical axis defined by orientation of $\mathbf{n}(\mathbf{r})$ and the phase retardation defined by the optical anisotropy of the LC and polar angle of the director. The resulting polarizing optical micrographs were obtained as a result of successive multiplication of Jones matrices corresponding to a polarizer, a series of thin LC slabs each equivalent to a phase retardation plate with spatially varying optical axis and retardation, and an analyzer. A two-dimensional polarizing optical image (see examples in Fig. 7b) was then obtained by performing such a Jones-matrix calculation for each pixel and then composing a two-dimensional texture with coordinate-dependent intensity analogous to the experimental images (see examples in Fig. 7a). To properly account for the achromatic nature of our experimental POM observations, we calculated these textures separately for three different wavelengths spanning the entire visible spectrum $(475,510$, and $650 \mathrm{~nm})$ and then superimposed them according to the experimental light source intensities at the corresponding wavelengths. This yielded achromatic polarizing optical micrographs analogous to the corresponding experimental images (Fig. 7a, b). Computer-simulated 3PEF-PM cross-sections were constructed for the director structures obtained as described above by first finding the coordinate-dependent angles $\psi$ between $\mathbf{n}(\mathbf{r})$ and the linear polarization of the probing laser light and then plotting the normalized signal intensity as $I_{3 \text { PEF-PM }}=\cos ^{6} \psi$ (note that this modeling disregards finite resolution effects, as well as various issues associated with scattering and defocusing of light in the birefringent medium). Despite the large number of simplifications and assumptions, all experimental and computer-simulated images of $\mathbf{n}(\mathbf{r})$-structures in chiral nematic samples closely match each other and strongly support our interpretation of experimental findings (Figs. 4, 5a, b, 7a, b).

Data availability. Additional schematics, images and videos are available in the Supplementary Information. Matlab and Fortran codes and both experimental and computational datasets generated and analyzed during the current study are available from the corresponding author on request.

Received: 18 April 2017 Accepted: 19 July 2017

Published online: 22 September 2017

\section{References}

1. Chaikin, P. M., Lubensky, T. C., Principles of Condensed Matter Physics (Cambridge Univ. Press, 1995).

2. Marchetti, M. C. et al. Hydrodynamics of soft active matter. Rev. Mod. Phys. $\mathbf{8 5}$ 1143 (2013).

3. Sanchez, T., Chen, D. T. N., DeCamp, S. J., Heymann, M. \& Dogic, Z. Spontaneous motion in hierarchically assembled active matter. Nature 491, 431-434 (2012).

4. Thampi, S. P., Golestanian, R. \& Yeomans, J. M. Velocity correlations in an active nematic. Phys. Rev. Lett. 111, 118101 (2013).

5. Giomi, L., Bowick, M. J., Ma, X. \& Marchetti, M. C. Defect annihilation and proliferation in active nematics. Phys. Rev. Lett. 110, 228101 (2013).

6. Rößler, U. K., Bogdanov, A. N. \& Pfleiderer, C. Spontaneous skyrmion ground states in magnetic metals. Nature 442, 797-801 (2006).

7. Romming, N. et al. Writing and deleting single magnetic skyrmions. Science 341, 636-639 (2013).

8. Yu, X. Z. et al. Real-space observation of a two-dimensional skyrmion crystal. Nature 465, 901-904 (2010).

9. Fert, A., Cros, V. \& Sampaio, J. Skyrmions on the track. Nat. Nanotechnol. 8, 152-156 (2013).

10. Nagaosa, N. \& Tokura, Y. Topological properties and dynamics of magnetic skyrmions. Nat. Nanotechnol. 8, 899-911 (2013).

11. Woo, S. et al. Observation of room-temperature magnetic skyrmions and their current-driven dynamics in ultrathin metallic ferromagnets. Nat. Mater. 15, 501-506 (2016).

12. Krause, S. \& Wiesendanger, R. Skyrmionics gets hot. Nat. Mater. 15, 493-494 (2016).

13. Skyrme, T. A unified field theory of mesons and baryons. Nuclear Phys. 31 , 556-569 (1962).

14. Smalyukh, I. I., Lansac, Y., Clark, N. A. \& Trivedi, R. P. Three-dimensional structure and multistable optical switching of triple-twisted particle-like excitations in anisotropic fluids. Nat. Mater. 9, 139-145 (2010).

15. Ackerman, P. J., Trivedi, R. P., Senyuk, B., van de Lagemaat, J. \& Smalyukh, I. I. Two-dimensional skyrmions and other solitonic structures in confinementfrustrated chiral nematics. Phys. Rev. E 90, 12505 (2014).

16. Ridley, A., Peckham, M., Clark, P., Cell Motility: From Molecules to Organisms. (Wiley, 2005).

17. Palagi, S. et al. Structured light enables biomimetic swimming and versatile locomotion of photoresponsive soft microrobots. Nat. Mater. 15, 647-653 (2016).

18. Trivedi, R. P., Klevets, I. I., Senyuk, B., Lee, T. \& Smalyukh, I. I. Reconfigurable interactions and three-dimensional patterning of colloidal particles and defects in lamellar soft media. Proc. Natl Acad. Sci. USA 109, 4744-4749 (2012).

19. Lee, T., Trivedi, R. P. \& Smalyukh, I. I. Multimodal nonlinear optical polarizing microscopy of long-range molecular order in liquid crystals. Opt. Lett. 35, 3447-3449 (2010).

20. Chen, R. H., Liquid Crystal Displays: Fundamental Physics and Technology. (Wiley, 2011).

21. Ackerman, P. J. \& Smalyukh, I. I. Diversity of knot solitons in liquid crystals manifested by linking of preimages in torons and hopfions. Phys Rev X 7, 011006 (2017).

22. Ackerman, P. J., van de Lagemaat, J. \& Smalyukh, I. I. Hierarchical selfassembly and electrostriction of defect and skyrmion arrays in chiral nematic liquid crystals. Nat. Commun. 6, 6012 (2015).

23. Leonov, A. O., Dragunov, I. E., Rößler, U. K. \& Bogdanov, A. N. Theory of skyrmion states in liquid crystals. Phys. Rev. E 90, 042502 (2014).

24. Afghah, S., Selinger. J. V., Theory of helicoids and skyrmions in confined cholesteric liquid crystals. Preprint at http://arXiv.org/abs/1702.06896v1 (2017).

25. Guo, Y. et al. Cholesteric liquid crystals in rectangular microchannels: skyrmions and stripes. Soft Matter 12, 6312-6320 (2016).

26. Sutcliffe, P. Chiral ferromagnetic fluids: Let's twist again. Nature Mater 16, 392-393 (2017). 
27. Liu, Q., Senyuk, B., Tasinkevych, M. \& Smalyukh, I. I. Nematic liquid crystal boojums with handles on colloidal handlebodies. Proc. Natl Acad. Sci. USA 110, 9231-9236 (2013)

28. Senyuk, B. et al. Topological colloids. Nature 493, 200-205 (2013).

29. Leonov, A. O., Loudon, J. C. \& Bogdanov, A. N. Spintronics via nonaxisymmetric chiral skyrmions. Appl. Phys. Lett. 109, 172404 (2016).

30. Wilson, M. N. et al. Extended elliptic skyrmion gratings in epitaxial MnSi thin films. Phys. Rev. B 86, 144420 (2012).

31. Rybakov, F. N., Borisov, A. B. \& Bogdanov, A. N. Three-dimensional skyrmion states in thin films of cubic helimagnets. Phys. Rev. B 87, 094424 (2013).

32. Milde, $\mathrm{P}$. et al. Unwinding of a skyrmion lattice by magnetic monopoles. Science 340, 1076-1080 (2013).

33. Vanbrabant, P. J. M., Beeckman, J., Neyts, K., James, R. \& Fernandez, F. A. Effect of material properties on reverse flow in nematic liquid crystal devices with homeotropic alignment. Appl. Phys. Lett. 95, 151108 (2009).

34. Lapointe, C. P., Hopkins, S., Mason, T. G. \& Smalyukh, I. I. Electrically-driven multi-axis rotational dynamics of colloidal platelets in nematic liquid crystals. Phys. Rev. Lett. 105, 178301 (2010).

35. Ackerman, P. J., Mundoor, H., Smalyukh, I. I. \& van de Lagemaat, J. Plasmon-exciton interactions studied using co-trapped semiconductor and plasmonic nanoparticles. ACS Nano 9, 12392-12400 (2015).

36. Efrati, E. \& Irvine, W. T. M. Orientation-Dependent handedness and chiral design. Phys. Rev. X 4, 011003 (2014).

37. Ackerman, P. J. \& Smalyukh, I. I. Static three-dimensional topological solitons in fluid chiral ferromagnets and colloids. Nat. Mater 16, 426-432 (2017).

38. Zhang, Q., Ackerman, P. J., Liu, Q. \& Smalyukh, I. I. Ferromagnetic switching of knotted vector fields in liquid crystal colloids. Phys. Rev. Lett. 115, 097802 (2015).

39. Baraban, L. et al. Transport of cargo by catalytic Janus micro-motors. Soft Matter 8, 48-52 (2012).

40. Karpierz, M. A., Spatial Solitons in Liquid Crystals. (Springer, 1991).

41. Muzny, C. D. \& Clark, N. A. Direct observation of the brownian motion of a liquid-crystal topological defect. Phys. Rev. Lett. 68, 6 (1992).

42. Migler, K. B. \& Meyer, R. B. Solitons and pattern formation in liquid crystals in a rotating magnetic field. Phys. Rev. Lett. 66, 11 (1991).

43. Helfrich, W. Alignment-inversion walls in nematic liquid crystals in the presence of a magnetic field. Phys. Rev. Lett. 21, 22 (1968).

44. Smalyukh, I. I., Butler, J., Shrout, J. D., Parsek, M. R. \& Wong, G. C. L. Elasticity-mediated nematic-like bacterial organization in model extracellular DNA matrix. Phys. Rev. E 78, 030701(R) (2008).

45. Abbott, N. L. \& Velev, O. D. Active particles propelled into researchers' focus. Curr. Opin. Colloid Interf.e Sci. 21, 1-3 (2016).

46. Martinez, A. \& Smalyukh, I. I. Light-driven dynamic Archimedes spirals and periodic oscillatory patterns of topological solitons in anisotropic soft matter. Opt. Exp 23, 4591-4604 (2015).

47. Lavrentovich, O. D. Active colloids in liquid crystals. Curr. Opin. Colloid Interface Sci 21, 97-109 (2016).

48. van Oosten, C. L., Bastiaansen, C. W. M. \& Broer, D. J. Printed artificial cilia from liquid-crystal network actuators modularly driven by light. Nat. Mater. 8 , 677-682 (2009).
49. Sheetah, G. H., Liu, Q. \& Smalyukh, I. I. Self-assembly of predesigned optical materials in nematic codispersions of plasmonic nanorods. Optics Lett. 41, 4899-4902 (2016).

50. Smalyukh, I. I. et al. Electric-field-induced nematic-cholesteric transition and three-dimensional director structures in homeotropic cells. Phys. Rev. E 72, 061707 (2005).

51. Anderson, J. E., Watson, P. E., \& Bos, P. J., Liquid Crystal Display 3D Director Simulator, Software and Technology Guide (Artech House, 1999).

\section{Acknowledgements}

This research was supported by the National Science Foundation Grant DMR-1410735. We thank Noel Clark, Ghadah Sheetah, Hayley Sohn, Mykola Tasinkevych, Sumesh Thampi and Julia Yeomans for discussions, as well as Ghadah Sheetah and Hayley Sohn for assistance.

\section{Author contributions}

P.J.A., T.B. and I.I.S. contributed to all aspects of this work and wrote the manuscript. I.I.S. conceived and designed the project.

\section{Additional information}

Supplementary Information accompanies this paper at doi:10.1038/s41467-017-00659-5.

Competing interests: The authors declare no competing financial interests.

Reprints and permission information is available online at http://npg.nature.com/ reprintsandpermissions/

Publisher's note: Springer Nature remains neutral with regard to jurisdictional claims in published maps and institutional affiliations.

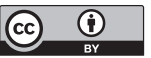

Open Access This article is licensed under a Creative Commons Attribution 4.0 International License, which permits use, sharing, adaptation, distribution and reproduction in any medium or format, as long as you give appropriate credit to the original author(s) and the source, provide a link to the Creative Commons license, and indicate if changes were made. The images or other third party material in this article are included in the article's Creative Commons license, unless indicated otherwise in a credit line to the material. If material is not included in the article's Creative Commons license and your intended use is not permitted by statutory regulation or exceeds the permitted use, you will need to obtain permission directly from the copyright holder. To view a copy of this license, visit http://creativecommons.org/ licenses/by/4.0/.

(C) The Author(s) 2017 